Christian Mann*

\title{
Könige, Poleis und Athleten in hellenistischer Zeit
}

https://doi.org/10.1515/klio-2018-0103

Zusammenfassung: Bei der Erforschung der politischen Kommunikation in der hellenistischen Welt haben die sportlichen Wettkämpfe bislang nicht die gebührende Aufmerksamkeit erfahren. Hier setzt der Aufsatz an, der aufzuzeigen versucht, wie Könige und Poleis die Agonistik als Kommunikationsraum nutzten, um Sieghaftigkeit zu demonstrieren, Loyalität zu bekunden und Status zu verhandeln. Konkret werden drei Phänomene in den Blick genommen: die Teilnahme der Könige an den Pferde- und Wagenrennen, die Partizipation der Könige am agonistischen Ruhm anderer und die Konstituierung und Ausrichtung von Agonen durch oder mit Bezug auf Könige. Dabei werden sowohl allgemeine Muster als auch spezifische Strategien einzelner Könige und Dynastien sichtbar.

Summary: Specialists for political interaction in the Hellenistic world have neglected the athletic competitions so far. This article aims to show how kings and poleis used the agones as a platform to demonstrate victoriousness, to declare loyalty and to negotiate status. The study covers three phenomena: the participation of kings in the horse and chariot races, the transfer of athletic fame from other victors to the kings, and the foundation and organization of agones by or with reference to the kings. Universal patterns are analyzed as well as specific strategies of some kings and dynasties.

Keywords: Hellenismus, Agonistik, politische Kommunikation, Monarchie, Polis

\section{Fragestellung und Forschungsstand}

Seit den 1990er Jahren hat sich die Erkenntnis, daß die griechische Polis keineswegs 338 v. Chr. auf dem Schlachtfeld von Chaironeia starb, sondern sich im Hellenismus einer ungebrochenen Vitalität erfreute, allgemein durchgesetzt. ${ }^{1}$ Damit

1 Die früheste und gewichtigste Stimme war diejenige Louis Roberts (Robert 1969, 42: „La cité grecque n'est pas morte à Chéronée, ni sous Alexandre, ni dans le cours de toute l'époque hellé-

*Corresponding author: Christian Mann, Universität Mannheim, Philosophische Fakultät, Historisches Institut, Mannheim, Deutschland, E-mail: mann@mail.uni-mannheim.de 
einher ging nicht nur ein veränderter Blick auf die politische, gesellschaftliche und architektonische Entwicklung der hellenistischen Poleis, ${ }^{2}$ sondern auch eine Neubewertung der politischen Kommunikation: Das Verhältnis zwischen Königen und Poleis sei nicht durch Befehl und Gehorsam, sondern durch Diplomatie und Aushandlung geprägt gewesen, und die Poleis seien, ob einzeln oder in bundesstaatlichen Organisationen, eigenständige Subjekte der griechischen Politik geblieben. Am weitesten ging in dieser Hinsicht John Ma, der in einem 2003 erschienenen Aufsatz das Zentrum-Peripherie-Modell, gemäß dem politische Macht und kulturelle Entwicklungen von den Königshöfen ausgingen, durch das Modell der „peer polity interaction“ ersetzt wissen wollte: Die hellenistische Welt habe man sich vorzustellen als ein Netzwerk von Einheiten, die einander auf Augenhöhe begegneten, eine gemeinsame Sprache teilten, sich gegenseitig beobachteten und voneinander lernten: „[...] the cities were structurally homologous, shared basic concepts, and were deeply aware of this shared culture. [...] thus the phenomenon itself attests a shared culture of emulation and imitation." ${ }^{\text {3 }}$ Ausdruck dieser dichten Vernetzung seien Gesandtschaften, wechselseitige Ehrungen und Geschenke.

Unbestritten blieb die ökonomische und militärische Übermacht der hellenistischen Könige, denen sich Ma in einem anderen, im selben Jahr publizierten Beitrag widmete: Er betont darin die Vielfalt des hellenistischen Königtums, womit er sowohl die Unterschiede zwischen den einzelnen Königen meint als auch besonders die Vielzahl der Rollen, die ein und derselbe Monarch auszufüllen hatte: Er mußte starker Heerführer sein, großzügiger Patron griechischer Poleis, unnachgiebiger Rächer an seinen Feinden, Bewahrer althergebrachter Traditionen, göttlicher Herrscher, um nur einige der Rollen zu nennen - Ma spricht von der „chameleon quality“ ${ }^{“ 4}$ hellenistischer Könige. Vor diesem Hintergrund zeigt er auf, wie man den Herrschaftsanspruch der Könige mit den heterogenen Erwartungen der Bevölkerung in Einklang zu bringen, wie man bei aller Diversität die Einheit des Reiches unter einem König zu gewährleisten versuchte; insbesondere analysiert er die Strategien, mit denen die Poleis in imperiale Strukturen eingebunden werden sollten. Die beiden Aufsätze Mas verhalten sich zueinander komplementär; sie betrachten die hellenistische Welt aus gegensätzlichen

nistique.“), wichtige frühe Beiträge zur Dekonstruktion der Verfallsthese waren Gauthier 1993, Gruen 1993 sowie die Aufsätze in Wörrle - Zanker 1995.

2 Für einen Überblick s. die im DFG-Schwerpunktprogramm „Die hellenistische Polis als Lebensform“ entstandenen Publikationen, vor allem Matthaei - Zimmermann 2009; iid. 2014; iid. 2015. 3 Ma 2003a, 11. Das Konzept der „peer polity interaction“ war ursprünglich in der Archäologie entwickelt worden (Renfrew - Cherry 1986).

4 Ma 2003b, 179. 
Perspektiven, sind aber durch ein gemeinsames Instrumentarium verbunden. Der Fokus liegt auf öffentlichen Inschriften als den besten Zeugnissen für den diplomatischen Austausch, die auf der Grundlage der Sprechakt-Theorie zugleich als Mittel und Ergebnis von Aushandlungsprozessen analysiert werden. ${ }^{5}$

Auf die Agonistik kommt Ma nur am Rande zu sprechen. Dies ist im Rahmen breit angelegter konzeptioneller Aufsätze nicht zu kritisieren, aber doch symptomatisch: Sport wird in den Forschungen zu den Königen und Poleis hellenistischer Zeit zumeist ignoriert oder lediglich im Kontext des Gymnasions wahrgenommen. ${ }^{6}$ Dies ist erstaunlich und bedauerlich, bildete doch die Agonistik bekanntermaßen einen wichtigen Kommunikationsraum, in dem Identitäten sichtbar, Konkurrenz zwischen einzelnen und zwischen Poleis ausgetragen, Ruhm und Prestige gewonnen, verloren und verhandelt wurden. Und die Agonistik verlor ihre Bedeutung in hellenistischer Zeit nicht: Zwar postulierte die ältere Forschung für diese Epoche einen Niedergang, aber wie für die Polis ist auch für die Agonistik die Dekadenzthese längst passé. ${ }^{7}$ Die traditionellen Agone erfreuten sich, zumindest bis zu den Wirren der Mithridatischen Kriege, ungebrochener Beliebtheit, und neue Agone wurden gegründet. Auch blieb die Begeisterung für Athleten und deren Bedeutung als Identifikationsfiguren ungebrochen, wovon zahlreiche Inschriften zeugen: Die „Database of Hellenistic Athletes“8 verzeichnet über 2.000 überlieferte Sieger in gymnischen und hippischen Wettkämpfen, der größte Teil von ihnen ist epigraphisch überliefert.

Vor diesem Hintergrund erscheint es lohnend, die Aushandlungsprozesse in der hellenistischen Welt einmal aus der agonistischen Perspektive $\mathrm{zu}$ untersuchen. Dies ist bislang nicht geschehen; zwar haben die im berühmten „Neuen Poseidipp“ enthaltenen Hippika einiges Interesse hervorgerufen, die historische Kontextualisierung der Epigramme ist jedoch noch nicht weit gediehen. Die

5 So auch schon Ma 1999, 19-22. Einen hervorragenden Überblick über die Forschungen zum hellenistischen Königtum liefert Wiemer 2017.

6 Bezeichnenderweise finden sich die Begriffe „Sport/sports“, „Athleten/athletes“ oder „Agonistik/agonistics“ weder als Lemmata im einschlägigen Lexikon (Schmitt 2005) noch in den Indizes angelsächsischer Companions zum Hellenismus (Erskine 2003; Bugh 2006). Zu sportlichem Training und Wettkämpfen im Gymnasion s. die Verweise in Kah - Scholz 2004.

7 Ältere Handbücher stellten die archaische und klassische Epoche - als angebliche Epoche des ,guten' griechischen Amateursports - ins Zentrum, während man den Hellenismus mit Professionalisierung und Niedergang verband (Gardiner 1930; Harris 1972); laut Pleket (1975, 51) wurde damit ein kleiner Bruder zum Niedergang des Römischen Reiches geschaffen. Pleket trug mit seinen epigraphischen Studien (1978; 2001; 2005) wesentlich zu einem neuen Verständnis der hellenistischen Agonistik bei; s. dazu Langenfeld 2009 und jetzt ausführlich Mann - Remijsen Scharff 2016.

8 http://mafas.geschichte.uni-mannheim.de/athletes. 
schmale Monographie von Di Nanni Durante bietet trotz des vielversprechenden Titels lediglich eine Zusammenstellung wichtiger Quellen, aber keine zusammenhängende Interpretation der hellenistischen Agonistik. ${ }^{9}$ Vor allem wurde bislang noch nicht das besondere Erkenntnispotenzial der Agonistik im Hinblick auf die Vielfalt des hellenistischen Königtums erkannt. Während die einzelnen Reiche sich in ihrer Größe, Wirtschafts- und Bevölkerungsstruktur markant voneinander unterschieden, so daß sich angesichts der ungleichen Voraussetzungen königliches Handeln kaum miteinander vergleichen läßt, waren die Wettkämpfe für alle gleichermaßen zugänglich. Die Agonistik bietet also die Möglichkeit, sowohl allgemeine Verhaltensmuster als auch spezifische königliche Strategien zu erkennen. Dies soll im Folgenden in drei Abschnitten geschehen: Zunächst werden die von Königen errungen Siege behandelt, anschließend die Teilhabe der Könige an agonistischen Erfolgen anderer und zuletzt die Konstituierung und Ausrichtung von Agonen durch oder mit Bezug auf die Könige analysiert.

\section{Könige als agonistische Sieger}

Von den drei Wettkampfkategorien der griechischen Agonistik waren die hippischen Disziplinen bei Alleinherrschern am beliebtesten. Der Grund liegt auf der Hand: Während für Erfolge bei den musischen und gymnischen Wettbewerben Talent und intensives Training vonnöten waren, gab bei den Pferde- und Wagenrennen die ökonomische Potenz den Ausschlag, da nicht Jockeys und Wagenlenker, sondern die Besitzer der Pferde als Sieger ausgerufen wurden. Es tat dem Prestige hippischer Siege keinen Abbruch, daß man sie nicht durch persönliche Performanz errang, und so legten viele Tyrannen und Könige großen Wert darauf, schnelle Pferde zu züchten oder zu kaufen, um mit ihnen Siegeskränze bei panhellenischen Wettkämpfen zu erringen. Ein frühes Beispiel stellt Kleisthenes von Sikyon dar, der in Olympia beim Rennen der Viergespanne erfolgreich war, ${ }^{10}$ ein besonders hervorstechendes Hieron von Syrakus, der bei den bedeutendsten Wettkämpfen in verschiedenen hippischen Disziplinen triumphierte und seine Siege durch die Epinikien Pindars und Bakchylides' sowie durch aufwendige Monumente in panhellenischen Heiligtümern verherrlichen ließ. ${ }^{11}$ Später errang Philipp II. von Makedonien in Olympia und Delphi mehrere Siege in Pferde- und Wagenrennen, die er offensichtlich für so bedeutsam hielt, daß er sie auf Münzen

9 Di Nanni Durante 2015; zu den Hippika s. u. Anm. 23 und 29.

10 Hdt. 6,126. Nach Moretti (1957, Nr. 96) fiel der Sieg in das Jahr 572 v. Chr.

11 Mann 2001, 236-273. 
prägen ließ. Auf diese Weise konnte er sowohl Reichtum und Macht zeigen als auch seine Zugehörigkeit zur griechischen Welt demonstrieren. ${ }^{12}$ Machtpolitisches Kalkül wird man neben dem Streben nach Ruhm - sofern sich beides überhaupt trennen läßt - als dominantes Motiv für die pferdesportlichen Aktivitäten griechischer Alleinherrscher annehmen dürfen.

Angesichts dieser Vorbilder und des gewaltigen Prestiges, das mit agonistischen Erfolgen verbunden war, sollte man eigentlich annehmen, daß alle hellenistischen Könige darum wetteiferten, in Olympia und anderen panhellenischen Wettkampfstätten Siege zu erringen. Diese Annahme kann man auch recht häufig lesen, beispielsweise schreibt Silvia Barbantani, eine ausgewiesene Expertin für hellenistische Siegesdichtung: „It is no wonder, then, that the most powerful Hellenistic dynasts took part in the main Greek games. "13 Auf der anderen Seite steht das Statement von Ioanna Kralli: „Participation in the Panhellenic games was normally not an option for a king. " ${ }^{14}$ Beide Aussagen sind in ihrer Allgemeinheit falsch, was - dies sei der Fairneß halber betont - den Autorinnen auch bewußt ist. Sie haben nur konträre Auffassungen davon, was als Regelfall und was als Ausnahme aufzufassen ist. Um zunächst die Fakten zu klären, sei auf die Tabellen verwiesen, die Sebastian Scharff auf der Grundlage der genannten „Database of Hellenistic Athletes“ erstellt hat: Aus diesen geht eindeutig hervor, daß sich nur für zwei hellenistische Herrscherdynastien ein wirklich starkes agonistisches Engagement nachweisen läßt: für Ptolemäer und Attaliden. ${ }^{15}$ Für die Seleukiden hingegen ist nur eine panathenäische Siegerliste zu nennen, die zwei Erfolge eines Sohnes des Antiochos IV. Epiphanes nennt; ${ }^{16}$ der Name des Siegers selbst ist auf dem Stein leider nicht erhalten. In Frage kommen Antiochos V. und Alexander Balas, Tracy und Habicht favorisieren mit guten chronologischen Argumenten die letztere Lösung. ${ }^{17}$ Für die Antigoniden gibt es überhaupt keine Belege für eine Teilnahme an den Pferde- und Wagenrennen der großen Agone.

12 Kyle 2007, 232: „King Philipp II (359-336) used sport and sanctuaries to legitimize his extension of power over Greece.“

13 Barbantani 2012, 46.

14 Kralli 2013, 150.

15 Scharff i. Dr., mit einer Liste der überlieferten agonistischen Siege hellenistischer Monarchen. Nützlich ist auch der Appendix II bei di Nanni Durante 2015, 71-115, mit einer Übersicht über einige Wettkämpfe und Teilnehmer; jedoch bleiben hier die Kriterien für die Materialauswahl unklar.

$16 \mathrm{IG} \mathrm{II}^{2}$ 2317, Z. $36 \mathrm{f}$. $47 \mathrm{f}$.

17 Tracy - Habicht 1991, 218; sie setzen die Siege in die Jahre 150/49 oder 146/5 v. Chr. Zu den Legitimationsstrategien des Alexander Balas s. jetzt Chrubasik 2016, 129-135. 161-169 (ohne Bezug zu den Panathenäensiegen). 
Man sollte diesen Befund nicht auf ein Überlieferungsproblem reduzieren. Schließlich ist die Quellenlage, bedenkt man die hohe Anzahl überlieferter Sieger, ja keinesfalls schlecht, und die Informationen verteilen sich auf verschiedene Quellengattungen. Zu nennen sind vor allem Siegerlisten, Inschriften auf Siegesmonumenten und die Beschreibung der Heiligtümer durch Pausanias, aber auch die Historiographie und andere literarische Genres. Diese breite Streuung macht den Befund robust gegen einzelne Zufallsfunde, auch schon vor der Publikation des Mailänder Papyrus überstrahlten die Ptolemäer hinsichtlich ihrer agonistischen Erfolgsbilanz die Seleukiden bei weitem. Auch die Annahme, teilgenommen hätten alle Könige, die Ptolemäer und Attaliden seien lediglich erfolgreicher gewesen als die anderen und hätten deshalb mehr Spuren in der Überlieferung hinterlassen, ist angesichts der guten Bedingungen für die Pferdezucht in den von Antigoniden und Seleukiden beherrschten Gebieten ${ }^{18}$ nur eine Notlösung: Wenn selbst der Numider Mastanabal Eingang in die Siegerlisten gefunden hat, ${ }^{19}$ sich dagegen für mächtige und äußerst dynamische Könige wie Philipp V. oder Antiochos III. keine Belege für ein hippisch-agonistisches Engagement finden lassen, dann sollten wir nach aller Plausibilität davon ausgehen, daß letztere eben keine Pferde zu den Wettkämpfen schickten. Unter methodischen Gesichtspunkten jedenfalls wäre es die schlechtere Option, nicht überlieferte Siege einfach zu postulieren; besser ist es, Erklärungen für den gegenwärtigen Befund zu finden, mit aller Vorsicht und dem Bewußtsein, daß künftige Inschriften- und Papyrifunde das Bild beträchtlich verändern können. Es sind also zwei Fragen zu bearbeiten: Warum beteiligten sich manche Monarchen massiv an den Pferde- und Wagenrennen, und warum beteiligten sich andere Monarchen nicht? Gerade der Verzicht bedarf angesichts der Bedeutung, die der Sieghaftigkeit für die Legitimität hellenistischer Monarchen zukam, ${ }^{20}$ einer Erklärung.

Zunächst zur ersten Frage und zu den Ptolemäern, deren Siegesserie bei panhellenischen Wettkämpfen beeindruckend ist: ${ }^{21}$ Sie begann mit Ptolemaios I. Soter, erreichte ihren Höhepunkt unter Ptolemaios II. Philadelphos und setzte sich im 2. und 1. Jahrhundert v. Chr. fort. ${ }^{22}$ Außer der bloßen Menge der Siege ist

18 Die Antigoniden verfügten mit Thessalien, die Seleukiden mit den persischen Kernlanden über hervorragende Gebiete für die Pferdezucht (vgl. Hdt. 7,196).

19 IG II $^{2} 2316$, Z. 41-44.

20 Grundlegend dazu Gehrke 2013 (1982).

21 Übersichten bei van Bremen 2007, 363 f.; Remijsen 2009, 101-106; Di Nanni Durante 2015, 35-38; Scharff i. Dr.

22 Ptolemaios I.: Siege mit dem Viergespann in Olympia (Poseidipp. ep. 78,3f.) und mit dem Zweigespann der Fohlen in Delphi (Paus. 10,7,8); Ptolemaios II.: Siege mit dem Viergespann in Olympia (Poseidipp. ep. 88); Ptolemaios V.: Sieg mit dem Wagen bei den Panathenäen (IG II ${ }^{2}$ 2314, Z. 40-42); Ptolemaios VI.: Siege bei den Panathenäen mit dem Zweigespann und im Foh- 
auffällig, daß neben den Königen selbst auch Personen in ihrem Umfeld Pferde und Wagen zu den großen Rennen schickten: Königinnen, hohe höfische Würdenträger und mit Belistiche sogar eine Mätresse des Ptolemaios II. ${ }^{23}$ Und schließlich setzten die Ptolemäer nicht nur mit der Anzahl ihrer Siege, sondern auch mit deren Repräsentation Maßstäbe: Kallimachos revitalisierte die Gattung des Epinikion im ptolemäischen Kontext, und sechs der 18 in der Sektion „Hippika“ im neuen Poseidippos-Papyrus erhaltenen agonistischen Epigramme sind ptolemäischen Siegern gewidmet. ${ }^{24}$ Auch die Selbstdarstellung als siegreicher Pankratiast ist in diesem Kontext zu erwähnen. ${ }^{25}$ Es ist offensichtlich, daß man am Hof von Alexandria agonistischen Erfolgen eine größere Bedeutung zumaß als andernorts. Die Frage ist nur, weshalb.

Mit ägyptischen Vorbildern kann man das Phänomen nicht begründen, darin ist sich die Forschung einig. Zwar ließen sich Pharaonen des Neuen Reichs gerne auf Streitwagen abbilden, ${ }^{26}$ doch eine Tradition der Pferde- oder Wagenrennen ist für das vorhellenistische Ägypten nicht bezeugt, und die Repräsentation der Erfolge erfolgte allein in griechischen Medien und richtete sich deshalb an ein griechisches Publikum, nicht an die indigene ägyptische Bevölkerung. Die von Kralli vorgelegte geostrategische Erklärung, gemäß der die Ptolemäer durch ihre Teilnahme an Wettkämpfen auf der Peloponnes ihren Einfluß in dieser Region hätten stärken wollen, ${ }^{27}$ greift zu kurz: Erstens war ihr agonistisches Engagement nicht auf die Peloponnes beschränkt, sondern schloß Delphi und Athen mit ein die Konzentration von Siegen auf der Peloponnes ist schlicht darin begründet, daß drei der vier großen Wettkämpfe dort stattfanden. Zweitens stand mit der Gattung der monumentalen Weihgeschenke ein probateres Mittel zur Verfügung, um Macht, Reichtum und Großzügigkeit in panhellenischen Heiligtümern zu

lenrennen (SEG XLI 115, III, Z. 31-33; IG II² 2316, Z. 44-46); Ptolemaios XII.: Sieg bei den Basileia in Lebadeia mit dem Viergespann (Manieri 2009, Leb. 11, A, Z. 18-19).

23 Berenike I. (Poseidipp. ep. 78. 82. 88); Arsinoë II. (Poseidipp. ep. 78); Berenike II. (Kall. Victoria Berenices); Kallikrates (Poseidipp. ep. 74); Sosibios (Kall. fr. 384); Belistiche (P.Oxy. XVII 2082; Paus. 5,8,11). Die Identität der von Poseidippos in den Epigrammen 78, 79 und 82 gefeierten Berenike ist nicht zweifelsfrei zu bestimmen, in Frage kommen Berenike II. und Berenike „die Syrerin“. Aus dem umfangreichen Schrifttum zu dieser Frage seien genannt: Bennett 2005; Thompson 2005 (für Berenike „die Syrerin“); Huß 2008; Clayman 2012 (für Berenike II.).

24 Kallimachos Fr. 384 (Pfeiffer); SH 254-268C. Poseidipp ep. 74. 78. 79. 82. 87. 88, möglicherweise auch 80 und 81.

25 Trotz der Einwände von Thomas (1999) sprechen die stärkeren Argumente für die traditionelle Einordnung der „Pankratiastengruppe Istanbul“ in den ptolemäischen Kontext (Kunze 2002, 155-168 mit Abb. 68-73).

26 Decker 1987, 48-63, mit Beispielen.

27 Kralli 2013. 
demonstrieren, und auf dieses Mittel griffen die Ptolemäer ebenso wie andere hellenistische Dynastien zurück. Drittens war die Peloponnes auch für die Antigoniden ein Gebiet, auf das sich ihr Interesse richtete; wenn man die geostrategische Erklärung für das ptolemäische Engagement akzeptiert, wäre die Abwesenheit der Antigoniden von den hippischen Wettkämpfen in Olympia und Nemea umso schwerer zu begründen. Und viertens ist der Ruhm agonistischer Siege gerade nicht auf eine konkrete Region bezogen, sondern entfaltet wie die Agone selbst eine panhellenische Wirkung.

Überzeugender sind neuere Ansätze, die von der Art und Weise ausgehen, wie die Ptolemäer ihre agonistischen Erfolge in Dichtung und Monumenten darstellen ließen, und Bezüge zu spezifischen Merkmalen der ptolemäischen Herrschaft analysieren. Dabei liegt das Augenmerk auf zwei Aspekten: auf der Konstruktion der ptolemäischen Familie als agonistischer Ruhmgemeinschaft und auf der Betonung der Zugehörigkeit zur griechisch-makedonischen Welt. ${ }^{28}$ Beide Motive seien an einem der neuen Poseidippos-Epigramme dargestellt:

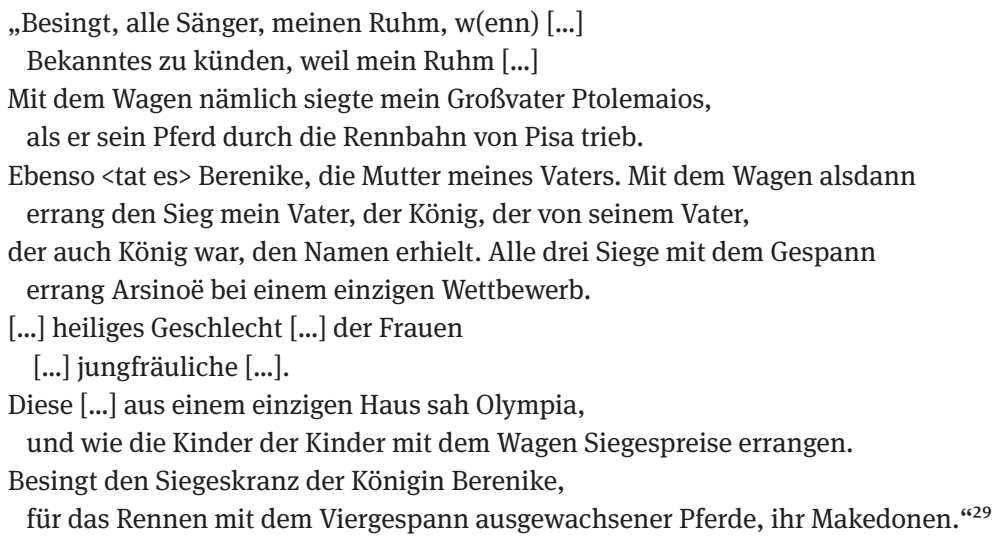

28 Kainz 2016; Scharff i. Dr.

29 Poseidipp. ep. 78 Austin - Bastianini 2002 (P.Mil.Vogl. VIII 309, XII 20-33):

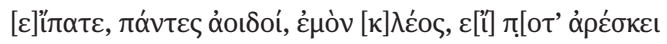

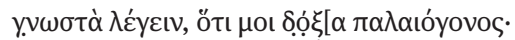

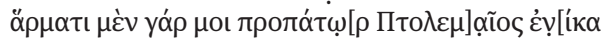

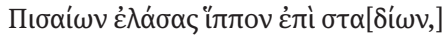

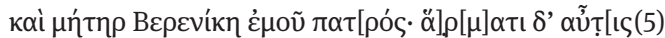

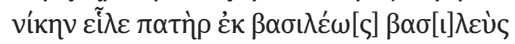

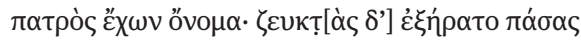

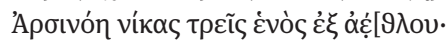

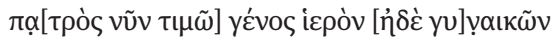

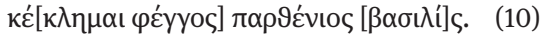


Unabhängig von der Frage, um welche Berenike es sich bei der Empfängerin des Epigramms handelt (s. Anm. 23), liegt die Stoßrichtung dieser Zeilen klar zutage: Sie zielen darauf ab, die Laudanda in eine familiäre Tradition einzuordnen: Berenike war beim Rennen der Viergespanne in Olympia erfolgreich gewesen, genau wie zuvor ihr Großvater, ihre Großmutter, ihr Vater und ihre Mutter, letztere hatte dazu noch zwei weitere Siege errungen. ${ }^{30}$ Es ist in der agonistischen Siegesdichtung zwar nichts Ungewöhnliches, daß auch die Erfolge von engen Verwandten genannt werden, verwiesen sei hier auf die Epinikien für Athleten aus Ägina ${ }^{31}$ oder auf das Daochos-Weihgeschenk. ${ }^{32}$ Doch die Präsentation einer lückenlosen Siegesserie über drei Generationen hinweg findet kein Vorbild, sie verweist auf bekannte Spezifika der ptolemäischen Dynastie: die starke Stellung der Frauen, die Geschwisterehe und den Dynastiekult. ${ }^{33}$

Ein zweiter auffälliger Aspekt ist die Betonung der makedonischen Identität in diesem wie in anderen Epigrammen Poseidipps für Ptolemäer. ${ }^{34}$ Nach Sabine Müller richtet sich Poseidipp an ein makedonisches Publikum und verweist auf seine eigene makedonische Heimat, ${ }^{35}$ doch eine solche biographische Erklärung ist wenig überzeugend. Daß nicht der Dichter, sondern die Auftraggeber für die Verweise auf makedonische Abstammung ausschlaggebend waren, zeigt der Vergleich mit anderen Gattungen agonistischer Siegesrepräsentation. Pausanias macht bei der Beschreibung der Siegerstatuen von Olympia eine merkwürdige Bemerkung: „Direkt neben Damiskos steht ein Mann, dessen Namen sie nicht angeben. Es ist eine Weihung des Ptolemaios, Sohn des Lagos. In der Inschrift

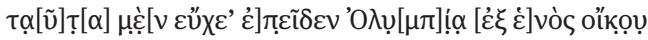

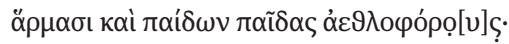

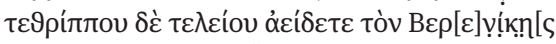

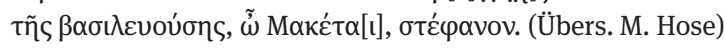

Die Ergänzungsvorschläge im griechischen Text folgen der editio minor (Austin - Bastianini 2002), für eine Diskussion s. auch Bastianini - Gallazzi 2001, 90 f. 207 f.; Hose 2015, 299-303. Auf der Homepage des „Center for Hellenic Studies“ werden regelmäßig aktuelle Forschungsübersichten publiziert (https://chs.harvard.edu/CHS/article/display/1343).

30 Zwischen der Abschaffung der apene, des Rennens der Maultiergespanne, 444 v. Chr. (Paus. 5,9,2) und der Einführung der synoris polon 264 v. Chr. (Paus. 5,8,11) fanden bei den Olympischen Spielen nur drei Wagenrennen statt: mit Vier- und Zweigespannen erwachsener Pferde sowie mit von Fohlen gezogenen Viergespannen.
}

31 Mann 2001, 200-202. $214 \mathrm{f}$.

32 Ebert 1972, Nr. 43-45. Für diesen Hinweis danke ich Anja Klöckner (Frankfurt).

33 Ausführlich dazu Kainz 2016, 335-343. Für Forschungspositionen zur Funktion des Dynastiekults und den Motiven, die Ptolemaios II. zur Heirat mit seiner Schwester Arsinoë bewogen, s. Müller 2009, 85-155; Carney 2013, 65-105.

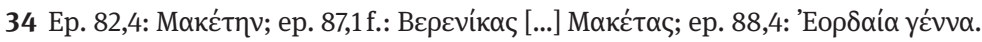
35 Müller 2009, 212. 
nennt Ptolemaios sich einen Makedonen, obwohl er doch in Ägypten herrschte. “36 Es ist wenig überraschend, daß Ptolemaios sich nicht König von Ägypten nennt, da das hellenistische Königtum nicht territorial gebunden war. Auffällig bleibt aber der systematische Verweis auf die makedonische Herkunft der Ptolemäer, und zwar in verschiedenen Gattungen der agonistischen Repräsentation für verschiedene Angehörige der Familie. Auch dies ist vor dem Hintergrund der spezifischen Situation dieser Dynastie zu verstehen. So vorteilhaft Ägypten als Kernraum der ptolemäischen Herrschaft aufgrund seines Reichtums auch war, es hatte den Nachteil, daß es aus der Perspektive der ,alten' griechischen Welt mit negativen Stereotypen besetzt, daß seine Zugehörigkeit zur griechischen Welt nicht eindeutig war. ${ }^{37}$ Dabei geht es weniger um die Frage, welchen konkreten Einfluß die indigene Bevölkerung auf die Herrscher in Alexandria hatte, sondern um den Exklusionsdiskurs. Dieser konnte gerade auf dem Feld der Agonistik aktiviert werden, wie eine bei Polybios überlieferte Anekdote zeigt: Im Finale des olympischen Faustkampfs 216 oder 212 v. Chr. standen sich Kleitomachos aus Theben, der Titelverteidiger und herausragende Kampfsportler seiner Zeit, und der bis dahin unbekannte Boxer Aristonikos aus Ägypten gegenüber. Als die Zuschauer für den Außenseiter Partei ergriffen,

„[...] soll Kleitomachos etwas zurückgetreten sein, kurz Atem geschöpft haben und sich dann an das Publikum mit der Frage gewandt haben, was sie sich eigentlich dabei dächten, daß sie für Aristonikos Partei nähmen und ihn nach Kräften moralisch unterstützten; ob sie etwa zweifelten, daß er den Kampf anständig führe, oder vergessen hätten, daß er, Kleitomachos, für den Ruhm der Griechen, Aristonikos für den des Königs Ptolemaios einstehe; ob sie es lieber sehen würden, daß ein Ägypter bei den Olympischen Spielen die Griechen besiege und den Kranz davontrage, oder daß ein Thebaner, ein Boioter als Sieger über alle Männer im Faustkampf ausgerufen werde.““38

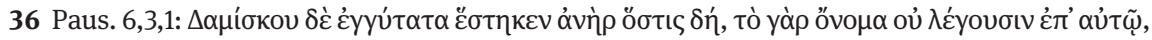

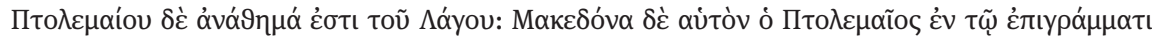

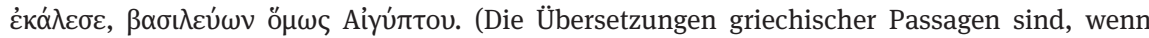
nicht anders angegeben, meine eigenen). Zur Betonung der makedonischen Abstammung durch Ptolemaios I. s. Bearzot 1992.

37 Erskine 2013, 344-352.

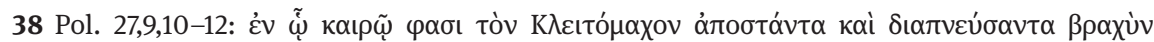

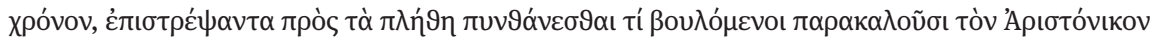

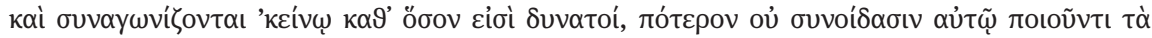

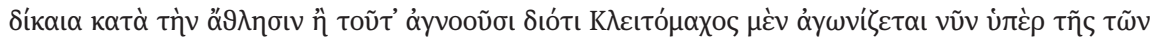

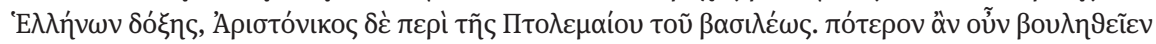

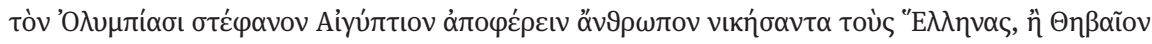

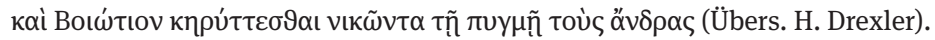

Laut Polybios zeigte die Ermahnung des Kleitomachos Wirkung, da das Publikum in der Folge ihn selbst und nicht mehr seinen Kontrahenten anfeuerte. 
In dieser Ansprache grenzt Kleitomachos seinen Kontrahenten gleich in zweierlei Hinsicht von sich (und dem Publikum) ab: die erste Grenzlinie verläuft zwischen einem Polisbürger und einem Königsgünstling, die zweite zwischen einem Griechen und einem Ägypter. Die zweite Differenz ist paradox, da die Olympischen Spiele nur Griechen offenstanden. Wer „Grieche“ war und wer nicht, war allerdings keine reine Frage der Abstammung, sondern kulturell definiert. Deshalb sollte man aus der Passage nicht den Schluß ziehen, Aristonikos sei ein indigener Ägypter gewesen, schließlich hatte man gegen seine Teilnahme keine Einwände erhoben. Auch wenn die Authentizität der Episode fraglich ist - Kleitomachos wird rein akustisch kaum Tausende von Zuschauern erreicht haben können, und angesichts der opulenten ptolemäischen Bauten in Olympia wird man nicht alle Zuschauer so leicht gegen diese Dynastie vereinnahmt haben können -, Faktum ist, daß Polybios einen sowohl ethnisch als auch politisch grundierten Exklusionsdiskurs bei den Olympischen Spielen für prinzipiell möglich hielt. Vor diesem Hintergrund kann man die agonistische Aktivität der Ptolemäer und die Zurschaustellung ihrer Erfolge auch dadurch motiviert sehen, daß sie - wie schon Philipp II. - ihre Zugehörigkeit zur griechischen Welt dokumentieren wollten; sie nutzten damit einen Kommunikationsraum, der wie kein anderer als Domäne der Griechen in Absetzung von den Barbaren galt. ${ }^{39}$ Die Ptolemäer sahen auf dieser Ebene offensichtlich einen größeren Legitimierungsbedarf als andere Dynastien.

Soviel zu den Ptolemäern. Die Hintergründe der attalidischen Siege seien hier nur gestreift, da Sebastian Scharff diese jüngst überzeugend analysiert hat: ${ }^{40}$ Auffällig ist vor allem die Konzentration der hippischen Aktivität auf Athen - sieben der acht überlieferten attalidischen Siege in Pferde- und Wagenrennen wurden bei den Panathenäen errungen - und die Zurschaustellung brüderlicher Gemeinschaft: Bei den Panathenäen des Jahres 178/77 v. Chr. errangen König Eumenes II. und seine Brüder Attalos, Philetairos und Athenaios jeweils einen Sieg. ${ }^{41}$ Auch hier läßt sich das agonistische Profil gut mit der Politik und Selbstdarstellung der Dynastie in Einklang bringen.

Die Beispiele der Ptolemäer und Attaliden machen die Frage, warum andere Könige die Bühne der panhellenischen Agone nicht nutzten, umso drängender. Da es mit historischen Methoden bekanntlich schwer zu erklären ist, warum Personen etwas nicht taten, sind die folgenden Überlegungen sehr hypothetisch. Zunächst ist auf Alexander den Großen zu verweisen. Dieser nahm, ganz im

39 Ein locus classicus ist Hdt. 2,91; zur Bedeutung der Agonistik für die griechische Identität s. Nielsen 2007, 12-21.

40 Scharff i. Dr.; s. auch Ebert 1972, Nr. 59; Shear 2007; Kertész 2013, 820-825; Di Nanni Durante 2015, $52 \mathrm{f}$.

41 IG II² 2314, Z. 83-90. 
Gegensatz zu seinem Vater, nicht an den panhellenischen Agonen teil, obwohl es ihm sicherlich leichtgefallen wäre, konkurrenzfähige Pferde und Gespanne in die Hippodrome zu schicken. Der Grund für das fehlende Interesse steht hier nicht zur Debatte, ${ }^{42}$ sondern die Auswirkung auf das Agieren hellenistischer Könige: Diese strebten danach, sich als jeweils legitime Nachfolger Alexanders zu inszenieren; bei diesem Wettstreit um die glaubwürdigste Alexanderimitatio waren agonistische Erfolge nicht nötig.

Zweitens, und dies ist der wichtigere Punkt, waren Agone ein hochsensibles, ambivalentes Feld, auf welchem Siege keinesfalls überall und uneingeschränkt bejubelt wurden. Alkibiades reklamierte mit Verweis auf seinen Olympiasieg 416 v. Chr. eine Führungsrolle in Athen, doch Thukydides macht deutlich, daß er mit seiner hippischen Aktivität auch Anstoß erregte, wie dies zuvor schon Megakles passiert war. ${ }^{43}$ Leslie Kurke interpretiert die Epinikien als eine Gattung, mit der potenzielle Spannungen zwischen dem einzelnen Sieger und der Polisgemeinschaft kanalisiert werden sollten. ${ }^{44} \mathrm{Ab}$ dem 4 . Jahrhundert v. Chr. nahmen diese Spannungen ab, dafür finden sich Hinweise, daß bei den Agonen antityrannische Ressentiments geschürt werden konnten. Während der Olympischen Spiele von 388 v. Chr. machte der Vorschlag die Runde, die Zelte der Abgesandten von Dionysios I. von Syrakus zu stürmen, und Diodors Bericht hört man noch deutlich die Schadenfreude über den Mißerfolg des mächtigen Monarchen an, dessen Gespanne nach Unfällen ausschieden. ${ }^{45}$ Die Mehrzahl geht aus dem Text eindeutig hervor, und man wird davon ausgehen können, daß Dionysios auch bei der Qualität von Pferden und Wagenlenkern nicht gespart hatte, doch Niederlagen sind beim sportlichen Wettkampf, der aufgrund der Verdichtung von Entscheidungen auf einen kurzen Zeitraum und wenige Situationen mehr Raum für Kontingenz läßt als andere gesellschaftliche Felder, immer möglich. Auch wenn für hellenistische Könige vergleichbare agonistische Desaster nicht überliefert sind, das Risiko einer Niederlage war auch für sie stets vorhanden.

In diesem Zusammenhang sollte auch berücksichtigt werden, daß Sport mehr als andere Felder sozialer Interaktion sowohl Gleichheit als auch Hierarchie konstruiert: Hierarchie durch die Ausdifferenzierung in Gewinner und Ver-

42 Plutarch schreibt Alexander das Dictum zu, er wäre in Olympia angetreten, wenn alle seine Gegner Könige gewesen wären (Alex. 4,5; mor. 179d. 331b). Kyle (2007, 239 f.) vermutet als Hintergrund ein orientalisches Monarchieverständnis, wonach ein König sich nicht einem Wettkampf aussetze.

43 Thuk. 6,15f.; s. dazu Mann 2001, 86-113.

44 Kurke 1991.

45 Diod. 14,109; Lys. 33, hypothesis. Bei der ganz ähnlichen Episode, die Plutarch über Themistokles erzählt $(25,1)$, handelt es sich wohl um eine Dublette. 
lierer, Gleichheit durch den Umstand, daß der Wettkampf nach für alle gleichen Regeln abläuft, die Teilnehmer sich innerhalb der Grenzen des Wettkampfs als Gleiche anerkennen. Eine Demonstration von Überlegenheit hat deshalb das Potenzial, Anstoß zu erregen. Es werden sicherlich nicht alle Griechen begeistert gewesen sein, wenn die Ptolemäer jahrzehntelang fast alle Siegeskränze in den prestigeträchtigen Wagenrennen abräumten, wenn sogar die Mätresse des Ptolemaios II. in Olympia gewann. Daß wir hiervon nichts in der reichen öffentlichen Inschriftenproduktion finden, auf der unsere Kenntnis vor allem beruht, darf nicht verwundern, da die betreffenden Gattungen gerade Harmonie, Eintracht und Ausgleich betonen. Ebensowenig ist verwunderlich, daß uns die Quellen keinen expliziten Einblick in die Motive gewähren, die hellenistische Könige zur Teilnahme oder Nichtteilnahme an den Agonen veranlaßten. Und wenn hier spezifische Strukturen der ptolemäischen und attalidischen Herrschaft zur Begründung herangezogen wurden, so muß auch hinzugefügt werden, daß die dort genannten Motive auch auf andere Dynastien anwendbar sind: Die Seleukiden beispielsweise waren aufgrund ihres Herrschaftsgebiets ebenso wie die Ptolemäer dem Vorwurf ausgesetzt, eher barbarische als griechische Könige zu sein, und Barbantani hat auf anderen Gebieten der ,Kulturpolitik' enge Parallelen zwischen beiden Dynastien aufzeigen können. ${ }^{46}$ Daher hätten auch die Seleukiden versuchen können, durch agonistische Erfolge ihre Position in der griechischen Welt zu untermauern. Zwangsläufig waren die Entscheidungen für oder gegen eine Teilnahme keineswegs, hier ging es darum, die möglichen Motive für das eine wie für das andere aufzuzeigen.

Eine Möglichkeit, Sieghaftigkeit mit einer Hommage an eine bedeutende Polis zu verbinden, boten die Panathenäen in Athen. Diese hatten neben Disziplinen, die allen Griechen offenstanden, auch solche im Programm, an denen nur athenische Bürger teilnehmen durften. In fünf nachgewiesenen Fällen siegten Ptolemäer und Attaliden in diesen Disziplinen, die Siegerlisten verzeichnen sie als Angehörige der nach ihnen benannten Phylen Ptolemais bzw. Attalis. ${ }^{47}$ Das monarchische Element tritt hier zurück; durch ihr Antreten als Mitglieder einer attischen Phyle zeigten sie ihre Wertschätzung für Athen, dem trotz seiner geschwundenen politischen Macht immer noch die Funktion eines „Leuchtturms“ der griechischen Welt zukam. Gerade in einer Zeit, in der die charismatische Grundlage des hellenistischen Königtums durch die immer deutlicher sicht-

46 Erskine 2013, 353; Barbantani 2014.

47 Ptolemaios V. 182/81 v. Chr. (IG II² 2314, Z. 40-42); Ptolemaios VI. 162/61 v. Chr. (SEG XLI 115, III, Z. 31-33); Eumenes II. und Attalos II. 170/69 v. Chr. (SEG XLI 115, I, Z. 37 f. 47 f.); Eumenes II. 162/61 v. Chr. (SEG XLI 115, III, 23f.). S. dazu Tracy - Habicht 1991, 188 f. und ausführlich Shear 2007. 
bare Macht der Römer ins Wanken geriet, ${ }^{48}$ mag ein solcher integrativer Umgang mit der Agonistik ratsam gewesen sein.

\section{Könige als Teilhaber agonistischen Ruhms}

Könige mußten nicht notwendigerweise selbst an den Agonen teilnehmen, um agonistischen Ruhm zu erlangen. In diesem Abschnitt soll betrachtet werden, in welcher Weise sie an den Erfolgen anderer Athleten teilhaben konnten. Das erste Beispiel greift auf die oben angeführte Anekdote zum olympischen Faustkampf zurück: Der erwähnte Faustkämpfer Aristonikos, Gegner des Kleitomachos, wurde laut Polybios von Ptolemaios IV. Philopator persönlich gefördert:

„Gegen ihn (sc. Kleitomachos), der als unbesiegbar galt und dessen Ruhm durch die ganze Welt ging, soll König Ptolemaios, der seine Ehre darein setzte, diesen Ruhm zu zerstören, nach sorgfältigstem Training den Faustkämpfer Aristonikos, der für diese Sportart eine hervorragende Kondition zu haben schien, in den Ring gestellt haben.“499

Merkwürdig ist die Angabe, Ptolemaios’ Aktivität sei gegen Kleitomachos gerichtet gewesen, denn für eine Feindschaft zwischen dem König und dem thebanischen Athleten läßt sich kein schlüssiges Motiv finden. Eher ging es darum, daß der Ruhm eines von Aristonikos errungenen Olympiasiegs auch auf den König selbst abstrahle. Die Förderung eines talentierten Athleten zu diesem Zweck ist aufgrund von mehreren Argumenten sehr plausibel: Erstens hat Kostas Buraselis eine Häufung von gymnischen Siegern aus dem Ptolemäerreich konstatiert; in der Olympionikenliste der betreffenden Zeit finden sich weitere Athleten aus Ägypten, und eine Inschrift in Argos läßt sich am besten als Dank zweier Athleten an König Ptolemaios für die Unterstützung ihrer agonistischen Karriere interpretieren. ${ }^{50}$ Zweitens hat Sofie Remijsen in diesem Zusammenhang die von den

48 Dazu Gotter 2013.

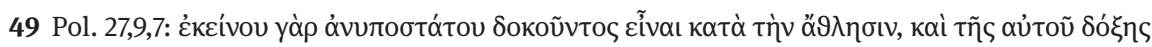

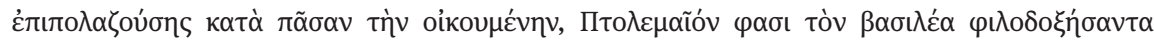

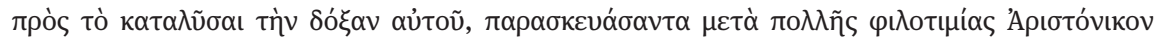

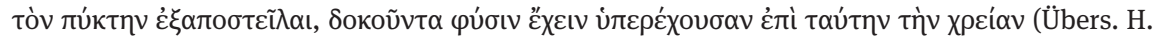
Drexler).

50 Buraselis 1993, 260. Bei den Olympioniken handelt es sich um Demetrios aus Alexandria im Stadionlauf 228 v. Chr. (Moretti 1957, Nr. 575), einen unbekannten Athleten aus Nibis 220 v. Chr. (Moretti 1957, Nr. 581) und Krates aus Alexandria im Stadionlauf 212 v. Chr. (Moretti 1957,

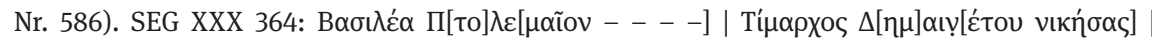

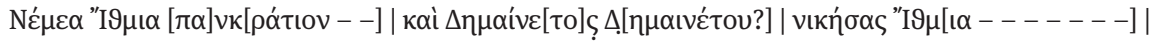


Ptolemäern an Athleten und Trainer verliehenen Vergünstigungen angeführt. ${ }^{51}$ Drittens kann man hinzufügen, daß Alleinherrscher bereits in vorhellenistischer Zeit Anstrengungen unternahmen, um an dem Ruhm von Olympioniken teilzuhaben: Gelon von Syrakus zog zwei der berühmtesten Athleten seiner Zeit, den Boxer Glaukos von Karystos und den Sprinter Astylos von Kroton, in seine Nähe, ${ }^{52}$ Abgesandte des Dionysios I. von Syrakus versuchten ein Jahrhundert später, den Vater eines Knabenboxers aus Milet zu bestechen, damit im Falle eines Sieges Syrakus als Polis ausgerufen werde. ${ }^{53}$ Vor diesem Hintergrund ist es gut denkbar, daß man nicht nur die Gewinnung fremder Athleten, sondern auch die Förderung von Athleten aus dem eigenen Herrschaftsbereich ins Auge faßte.

Ein weiteres Argument liefert der Umstand, daß seit dem frühen Hellenismus auch in anderen Konstellationen eine finanzielle Förderung talentierter Athleten nachweisbar ist: ${ }^{54}$ Im Zenon-Archiv findet sich ein Brief, in dem der Vorsteher des Gymnasions von Alexandria von den Fortschritten eines jungen Athleten namens Pythokles berichtet. Zenon hatte sich offensichtlich besorgt nach den Fortschritten seines Protegés erkundigt, wird aber in dem Brief beruhigt: Pythokles mache gute Fortschritte, künftige Siege seien zu erwarten, von denen auch Zenon selbst profitieren würde: „Ich hoffe, du wirst bekränzt werden!“555 Agonistischer Ruhm, so geht aus dieser Quelle eindeutig hervor, fällt auch auf den Mäzen. Daher ist es wenig erstaunlich, daß sich die Poleis ebenfalls um sportliche Talente kümmerten: So beschlossen die Ephesier im frühen 3. Jahrhundert v. Chr., einem Faustkämpfer namens Athenodoros die Kosten für das Training und die Wettkampfreisen zu bezahlen, zur Gegenfinanzierung wurde das ephesische Bürgerrecht an zwei Männer verkauft. Antragsteller war der Trainer gewesen, der darauf verwiesen hatte, daß Athenodoros bereits Siege errungen hatte und weitere, noch größere Erfolge in der Zukunft zu erwarten seien. ${ }^{56}$

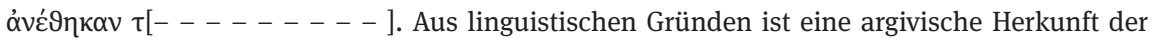
beiden Athleten unwahrscheinlich, eine Zuordnung nach Alexandria daher die plausibelste Lösung.

51 Remijsen 2009, 109.

52 Glaukos wurde als ,Vizetyrann' in Kamarina eingesetzt, Astylos ließ sich bei den Olympischen Spielen 484 und 480 v. Chr. als Syrakusaner ausrufen; s. dazu Mann 2001, 244-248.

53 Paus. 6,2,6f.

54 Ausführlich dazu Mann 2017.

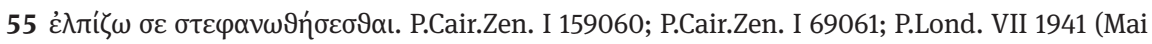
257 v. Chr.).

56 IEphesos 2005; dazu Pleket 2001, 186; Brunet 2003, 229. Bereits zuvor hatte Athenodoros, der offensichtlich Sohn eines Metöken war, bei den Nemeischen Spielen gesiegt und sich als Ephesier ausrufen lassen, wofür er mit dem Bürgerrecht belohnt wurde (IEphesos 1415). 
Die Vorstellung, daß der Ruhm erfolgreicher Athleten auf andere abstrahle, war also völlig gängig. Wenn reiche Mäzene und Poleis Geld ausgaben, um in Erwartung künftiger Siege junge Talente zu fördern, gibt es wenig Grund, Polybios' Angabe anzuzweifeln, daß Aristonikos' Karriere vom König unterstützt wurde. Philopator selbst errang keine Siege; einen Kursschwenk hin von einer persönlichen Teilnahme der Könige hin zu einer indirekten Partizipation an agonistischem Ruhm zu postulieren ist spekulativ, und bei der Annahme eines „sportlichen Leistungszentrums“ in Alexandria handelt es sich um einen Modernismus. ${ }^{57}$ Doch die Argumente für eine gezielte Förderung von Athleten sind stark, und das Motiv ist leicht zu entschlüsseln: Durch Siege von Athleten aus Ägypten konnte man unter Beweis stellen, daß im Kernland der Ptolemäer eben nicht nur Barbaren und degenerierte Griechen wohnten, sondern Männer, die sich in der griechischsten aller Aktivitäten zu bewähren wußten. ${ }^{58}$

Wie bei der persönlichen Teilnahme an den Agonen legten die Ptolemäer auch bei der Förderung von Athleten die größte Aktivität an den Tag. Doch auch auf die Seleukiden strahlte agonistischer Ruhm ab, wie ein Text zeigt, der alle drei im Titel des Aufsatzes genannten Kategorien - Könige, Poleis und Athleten - zusammenführt. Es handelt sich um eine Inschrift aus Teos, die zwei Ehrenbeschlüsse für Antiochos III. und Laodike sowie einen Brief des Seleukidenkönigs wiedergibt. ${ }^{59}$ Die Inschrift enthält zwei Ehrenbeschlüsse für Antiochos III. und Laodike sowie einen Brief des Seleukiden. Dieser hatte Teos, nachdem er diese und andere Poleis Kleinasiens den Attaliden entrissen hatte, wohlwollend behandelt, von Abgaben befreit und die asylia anerkannt. Im Gegenzug drückten die Bürger von Teos ihre Dankbarkeit aus, indem sie umfangreiche Ehrungen für König und Königin beschlossen, die sie auf einer beim Dionysostempel aufgestellten Inschrift festhielten. ${ }^{60}$ Der Text gibt wertvolle Aufschlüsse über den hellenistischen Herrscherkult und ist in diesem Kontext von der Forschung behandelt

57 Darauf hat Langenfeld (2009, 185-187) zurecht hingewiesen.

58 Buraselis 1993, 260. Hier findet sich auch die Überlegung, daß die agonistischen Erfolge in einer Zeit, als die finanziellen Schwierigkeiten der Ptolemäer eine energische Kriegführung in Griechenland nicht mehr zuließen, als Kompensation dienten. Eine ähnliche Erklärung liefert Scharff (i. Dr.) für die einzigen seleukidischen Siege des Alexander Balas.

59 Die Inschrift wurde 1963 entdeckt, Peter Herrmann erstellte zügig die editio princeps mit ausführlichen Kommentaren (Herrmann 1965). Piejko 1991 legte eine verbesserte Lesung vor, Korrekturen wurden von Herrmann selbst in SEG XLI 1003 vorgenommen.s. auch Rigsby 1996, 280-291; Kotsidu 2000, 346-355 KNr. 239; Aneziri 2003, 376-378 D3.

60 Im Text wird die persönliche Anwesenheit des Antiochos in Teos erwähnt, in Frage kommen die Jahre 204/3 und 197/6 v. Chr. Nach der Niederlage des Antiochos gegen die Römer fiel Teos wieder an die Attaliden, die Inschrift wurde abgeräumt und gelangte unter die Erde. 
worden, insbesondere von Angelos Chaniotis. ${ }^{61}$ Einer die Agonistik betreffenden Passage wurde allerdings bislang noch nicht die gebührende Aufmerksamkeit zuteil:

„Die Sieger in den Kranz-Agonen sollen bei ihrem feierlichen Einzug in die Stadt [...] von dem Tor sich zuerst in das Buleuterion begeben, sollen das Standbild des Königs bekränzen und gemäß der obigen Vorschrift opfern.“62

Vor der Interpretation sind Detailprobleme zu klären: Keine Probleme bereitet die Angabe der stephanitischen Agone, die bereits im 4. Jahrhundert v. Chr. als prestigeträchtigste Kategorie von Wettkämpfen nachgewiesen sind, ${ }^{63}$ doch über andere

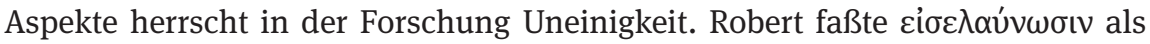
Einfahrt mit dem Wagen auf, das Verb bezeichnet aber gemäß Slater unspezifisch einen feierlichen Einzug in die Stadt. ${ }^{64}$ Nicht eindeutig geht aus dem Text hervor, ob die Personen einzeln an die Statue treten sollten oder, wenn es mehrere Sieger aus Teos gegeben hatte, als Gruppe. ${ }^{65}$ Ebenfalls nicht spezifiziert ist, für welche Sieger die Bestimmung galt: Chaniotis und Robert sprechen hier ausschließlich von Athleten, ${ }^{66}$ doch Teos war vor allem bekannt durch seine Sänger und Musiker - als Stadt des Dionysos war sie sogar Sitz eines thymelischen Technitenverbandes. ${ }^{67}$ Andererseits ist direkt vor der zitierten Passage von Epheben und Gymnasiarchen die Rede, ${ }^{68}$ was eher auf gymnische Sieger hindeutet. Die plausibelste Annahme ist, daß mit der Bestimmung sowohl gymnische als auch musische Sieger gemeint sind.

Trotz mancher offenen Fragen ist der in der zitierten Passage festgehaltene Akt hinreichend klar. Bürger von Teos, die bei einem stephanitischen Agon gesiegt hatten, sollten nach ihrer Rückkehr in die Heimat der „möglichst schönen Bronzestatue des Königs im Buleuterion"69 den gewonnenen Kranz aufsetzen und dem König opfern. Für Chaniotis sind bei diesem Akt zwei Aspekte bedeut-

61 Debord 2003, 286-289; Chaniotis 2005; id. 2007.

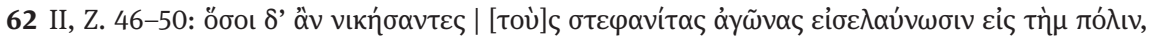

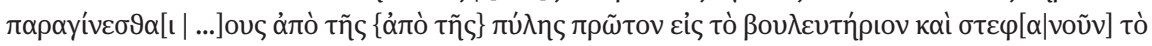

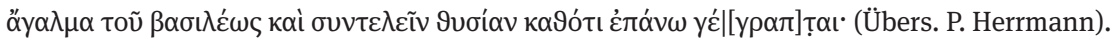

63 Dem. 20, 141; s. zu dieser Kategorie Remijsen 2011, mit einer Diskussion der älteren Literatur. 64 Robert 1984, 43; Robert - Robert 1989, 21; Slater 2013, 143 f.

65 Slater 2013, 144.

66 Robert 1984, 43; Chaniotis 2005, 197.

67 Strab. 14,1,29, der auch von einem späteren Umzug berichtet; s. dazu Aneziri 2003, 80-84. Zum Verhältnis zwischen Techniten und Teos ebd. 87-104.

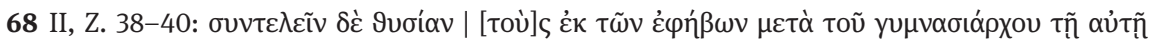

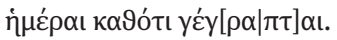

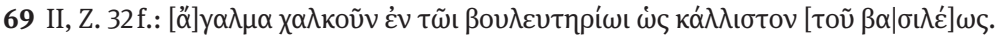


sam, „Sieghaftigkeit einerseits und Dankbarkeit andererseits““ ${ }^{70}$ Dies ist sicherlich richtig, man kann aber noch einen Schritt weitergehen: Der König hat am agonistischen Ruhm teil, er empfängt eine Art von Tribut, aber er empfängt ihn in einem Raum, der von der Polis kontrolliert wird, und nach Regeln, die von der Polis aufgestellt werden. Eine Abwertung agonistischer Siege, wie Ma sie in der Bestimmung sieht, ${ }^{71}$ vermag ich nicht zu erkennen. Vielmehr werden Athlet, Polis und König als eine agonistische Ruhmgemeinschaft konstruiert.

Das Symbol dieser Verbindung ist der Kranz, der auch in anderen Kontexten für einen Transfer athletischen Ruhms steht. In zahlreichen Texten finden sich Formulierungen, daß ein Sieger eine Person oder eine Gruppe bekränzt. ${ }^{72}$ Dies kann einen konkreten Akt bezeichnen wie im Fall von Teos, kann eindeutig metaphorisch gemeint sein oder unbestimmt - sprachlich ist zwischen konkreter und metaphorischer Bedeutung nicht zu entscheiden. Schon in den Epinikien Pindars ist die Ruhmübertragung per Kranz ein typisches Motiv, hier sollen drei epigraphische Zeugnisse angeführt werden:

„Er hat bei den Nemeen den Faustkampf der Knaben gewonnen, und indem er sich als Ephesier ausriefen ließ, hat er die Polis bekränzt."“73

„[...] als er die Kränze in die Heimatstadt hineinführte und die den Göttern zukommenden Opfer abhielt [...].“74

„Nachdem er in seiner eigenen Heimat in der Neuen Tragödie zum Wettkampf angetreten war und gesiegt hatte, bekränzte er unseren Demos.“75

Der erste Fall ist der häufigste: Ein Athlet bringt durch seinen Sieg, der auch als Sieg der Polis verstanden wird, der gesamten Bürgerschaft Ruhm. Der zweite Fall steht durch das Hereinbringen des Kranzes in die Stadt in der Verbindung mit Opfern dem Beispiel aus Teos nahe. Im dritten Fall geht es um den athenischen

70 Chaniotis 2005, 197.

71 Ma 1999, 222: „The message is perhaps that the king's power to benefit the city is superior to the symbolical advantages athletic victory brought to the city, or that the athletic contests are less important than the context to gain a city's gratitude through euergetism“.

72 S. dazu mit Beispielen: Robert - Robert 1989, 20-23; Rigsby 1996, 333 f.; Slater 2013, 144 Anm. 22. Ausführlich zum agonistischen Siegeskranz, seiner Verwendung und Bedeutung Blech 1982, 109-152.

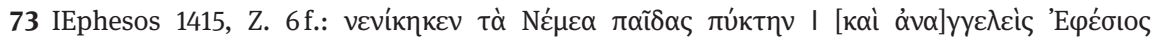

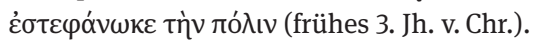

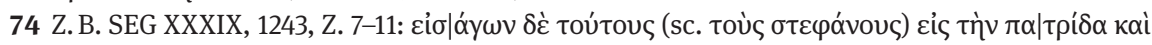

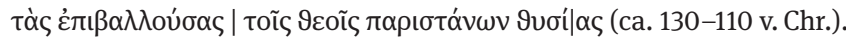

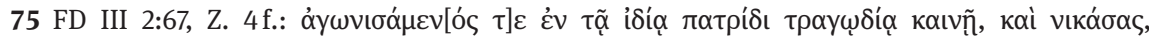

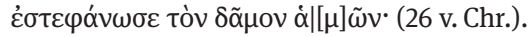


Tragöden Thrasykles, der seine guten Beziehungen zu Delphi - er war dort Hieromnemon - auch dadurch unter Beweis stellte, daß er sich bei der Siegerehrung der athenischen Dionysien als Delpher ausriefen ließ. Laut Kurke verfügten agonistische Siegeskränze in der Vorstellung der Griechen über göttliche Wirkkräfte; ${ }^{76}$ dies mag durchaus zutreffend sein, aber es ist kaum feststellbar, wie wichtig dieser magische Aspekt für die Betroffenen war. Hingegen ist die ganz irdische Bedeutung als Symbol athletischen Ruhms unzweifelhaft.

Die Bestimmung aus Teos fügt sich somit in den Umgang mit agonistischen Siegeskränzen ein, sie weist jedoch zwei Besonderheiten auf: Erstens wird die konkrete Übergabe des Kranzes durch den Athleten von der Polis beschlossen und damit verpflichtend gemacht, und zweitens ist der Empfänger des Kranzes nicht die Polisgemeinschaft, sondern der König. Hier verbindet sich die agonistische Bedeutung des Kranzes mit dessen Rolle im Herrscherkult. Bekanntlich spielen Kränze aller Art in der Kommunikation zwischen Poleis und Königen generell eine große Rolle. Für Antiochos III. berichtet eine Inschrift aus Herakleia am Latmos von Kranzgeschenken an die königliche Familie, bei denen anscheinend seitens der Polis nachgebessert wurde. ${ }^{77}$ Und auch in der Inschrift aus Teos taucht die Bekränzung der Königsstatue noch an einer anderen Stelle auf: „Der Priester des Königs soll dafür sorgen, daß auch sonst immer das Standbild des Königs mit einem der Jahreszeit entsprechenden Kranz geschmückt ist. “78 Diese Bekränzung findet, wenn die Priester ihrer Pflicht nachkommen, regelmäßig statt, die Bekränzung durch siegreiche Athleten hingegen hat zur Voraussetzung, daß es einen Sieg im Kranzagon gegeben hatte. Doch diese Schwäche der agonistischen Bekränzung wird durch ihre Stärke aufgewogen, nämlich daß ihr eine in der griechischen Welt anerkannte Leistung vorausgegangen ist; dieser Aspekt verleiht dem bei einem stephanitischen Agon gewonnenen Kranz seine besondere Kraft.

Die Bestimmung aus Teos ist ein Sonderfall, ${ }^{79}$ aber ein bezeichnender. Es geht hier um die Aushandlung von Status, die Rolle des Königs, der nicht nur an den materiellen, sondern auch an den symbolischen Ressourcen der Polis partizipieren soll, wird unterstrichen. Da agonistischer Ruhm für die Polis wichtig ist, bedeutet es keine Kleinigkeit, wenn der König daran durch einen offiziellen Beschluß beteiligt wird. Andererseits kann die Polis damit gut leben, denn der

76 Kurke 1993.

77 SEG XXXVII 859, Z. 1-6; dazu Wörrle 1988.

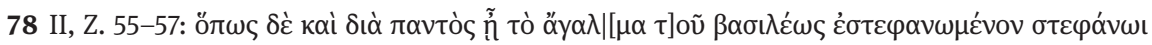

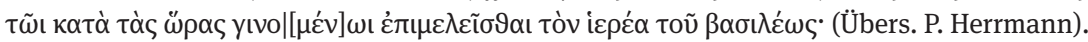

79 Möglicherweise enthält eine Inschrift aus Aigai (SEG LIX 1406, A, Z. 59-61; Malay - Ricl 2009, 39-47) eine ähnliche Bestimmung; s. dazu Scharff i. Dr. 
Akt vollzieht sich in ihrem Zentrum, und damit hat sie durch die vorgeschriebene Kranzübergabe auch selbst am agonistischen Ruhm teil, wie es in früherer Zeit durch die Aufführung von Epinikia geschah. ${ }^{80}$ Traditionelle Strukturen werden auf den König bezogen und dessen Macht gestärkt, ohne daß damit eine Unterwerfung der Polis einhergeht.

\section{Agone und die Verhandlung von Status}

Bislang war von agonistischen Siegern die Rede, im Folgenden geht es um die Agone selbst. Der Hellenismus sah eine Zunahme der Agone, doch die Annahme einer „explosion agonistique“ ${ }^{81}$ muß relativiert werden, denn sie berücksichtigt nur unzureichend die schon in klassischer Zeit hohe Anzahl sportlicher Wettkämpfe. ${ }^{82}$ Ohne Zweifel aber veränderten sich die Bedingungen für die Organisation: So kommt die neue Bedeutung bundesstaatlicher Strukturen in einem Beschluß des Akarnanischen Bundes zum Ausdruck, der in finanzielle Schwierigkeiten geratenen Stadt Anaktorion bei der Organisation der aktischen Spiele beizustehen; die Verteilung von Rechten und Pflichten zwischen Ethnos und Polis wird präzise geregelt. ${ }^{83}$

Alexander der Große selbst gründete keine Agone, ${ }^{84}$ doch für die hellenistischen Könige hatten Agone offensichtlich eine große Bedeutung, wie sich an Neugründungen zeigt. ${ }^{85}$ Der Bezug auf die Könige konnte auf ganz verschiedene Weise zum Ausdruck gebracht werden. Eine ebenso banale wie beliebte Möglichkeit war die Benennung des Agons nach dem König, so finden sich in hellenistischer Zeit zahlreiche Wettkämpfe mit Namen wie Ptolemaia, Eumeneia oder

80 Dazu Lattmann 2012.

81 Robert 1984, 38.

82 Nielsen 2016.

83 IG IX 12, 2, 583 (216 v. Chr.).

84 Zwar wird in der Forschungsliteratur regelmäßig postuliert, Alexander habe Herakleia in Tyros oder Basileia in Memphis gegründet (z. B. Moretti 1975, 106 f.; Koenen 1977, 29-31; Di Nanni Durante 2015, 11), doch dies geht aus den dafür zitierten Quellen (Arr. an. 2,24,6. 3,1,4. 3,5,2. 3,6,1; Plut. Alex. 29) gerade nicht hervor; bei den sportlichen Wettkämpfen auf dem Alexanderzug handelte es sich vielmehr um punktuelle Ereignisse, die nicht auf Wiederholung und RegelmäBigkeit ausgelegt waren. Zu dieser Thematik bereitet der Verfasser derzeit eine umfassende Studie vor.

85 Den Anfang machten bereits die Diadochen; für Antigonos Monophthalmos ist ein Agon auf Delos, für Seleukos Nikator in Troia sowie in Aigai bezeugt: IG XI,4 1036, Z. 4-10; IK Troia 31, Z. 9-12; SEG LIX 1406 A, Z. 49-51 (Malay - Ricl 2009, 39-47). 
Antigoneia. ${ }^{86}$ Doch es gab andere Optionen, beispielsweise konnte man auch mit dem Tag der Ausrichtung auf den König verweisen, wie das Ehrendekret für den Gymnasiarchen Menas zeigt:

„An den Geburtstagen des Königs richtete er monatlich Opfer zum Wohl des Volkes aus und veranstaltete Wettläufe für die Epheben und die Neoi, ebenfalls Wettkämpfe im Speerwurf und Bogenschießen und stiftete das Öl. Durch seine Ehrliebe wandte er die Jugend zum Training und zur Freude an den Übungen." ${ }^{\text {87 }}$

Die Bedeutung des Gymnasion für die hellenistische Polis kommt hier deutlich zum Ausdruck: Der Demos empfängt Opfer, die jungen Männer führen Wettkämpfe durch, und zwar sowohl in der klassischen sportlichen Disziplin des Wettlaufs als auch im Speerwurf und Bogenschießen, also Disziplinen mit betont militärischem Charakter, die es im Olympischen Programm nur im Kontext des Pentathlons bzw. gar nicht gab. Solche Kombinationen sind typisch für das hellenistische Gymnasion, ${ }^{88}$ sie verweisen auf die Wehrhaftigkeit der Polis. Dieses Ritual einer selbstbewußten Polis, die sich zu verteidigen weiß, ist nun aber gerade nicht gegen den König gerichtet, vielmehr wird es durch die Terminierung mit den königlichen Ansprüchen harmonisiert. ${ }^{89}$ Der König erscheint gleichsam als Garant und wohlwollender Beobachter der städtischen Demonstration militärischer Wehrhaftigkeit.

Ein anderes Beispiel: In einem Ehrendekret für den Kommandanten der örtlichen Garnison in Apollonia Salbake in Karien wird unter anderen folgende Ehrung beschlossen: „Und er (der Garnisonskommandant) soll jedes Jahr, wenn der gymnische Agon für König Seleukos gefeiert wird, auf den Ehrensitz gerufen

86 S. dazu den Appendix II von Di Nanni Durante 2015, mit zahlreichen Beispielen. Auch römische Feldherren wurden zu Namenspatronen von Agonen, z. B. Soteria kai Moukieia (IvO 327) oder Leukolleia (App. Mithr. 76).

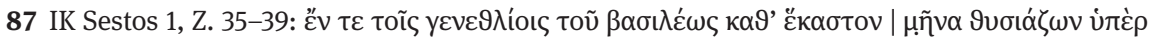

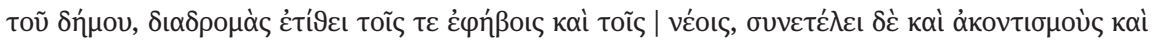

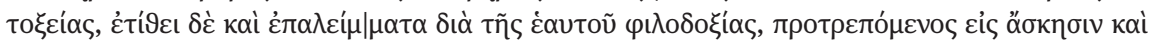

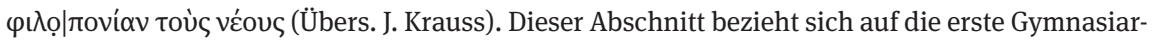
chie des Menas, als Attalos III. von Pergamon noch lebte; die Inschrift selbst entstand erst nach dessen Tod. Zum historischen Kontext s. den Kommentar von Krauss in IK Sestos, S. 14-29, zu den Aktivitäten des Menas. Albanidis 2015.

88 Zur Bedeutung militärischer Übungen im hellenistischen Gymnasion s. Chankowski 2004; Kah 2004. Kennell 2013, 227-229 hat darauf hingewiesen, daß der Begriff neoi in den zitierten Zeilen in zwei verschiedenen Bedeutungen gebraucht wird: zunächst gibt er eine Altersklasse im Gymnasion an, danach meint er allgemein die jüngeren Bürger der Polis.

89 Bei der Ansetzung der Wettkämpfe nach dem Geburtstag des Königs handelt es sich nicht um einen Einzelfall, s. die unten S. 468f. besprochenen Basileia. 
und mit einem goldenen Kranz bekränzt werden.“90 Die Inschrift stammt aus der Zeit Antiochos' III., bei dem genannten Seleukos handelt es sich wohl um Nikator, den Begründer der Dynastie. Bezeichnend ist, daß die jährliche Ehrung für den Garnisonskommandanten im Rahmen der Agone stattfindet, die auf den Begründer der seleukidischen Macht verweist. Die „hard power“ in Form der in der Polis stationierten Truppen und die „soft power“ der auf die Seleukidenherrscher verweisenden Wettkämpfe gehen hier Hand in Hand.

In Anbetracht der Größe vieler Königreiche gehörte es $\mathrm{zu}$ den wichtigsten imperialen Strategien, dem König in seinem Herrschaftsraum eine Präsenz jenseits der physischen Anwesenheit zu verschaffen. Die Agone boten hier eine gute Möglichkeit: Ein Ritual, das für die Polis von großer Bedeutung ist, wird mit dem Herrscher verknüpft, die Könige schreiben sich in die Polis ein. Damit wird ihre Macht zum Ausdruck gebracht, jedoch nicht in Form einer direkten Herrschaft über die Polis, denn der König oktroyiert schließlich keine neuartigen Strukturen auf. Kein Grieche hätte die Abhaltung von Agonen mit Königen oder gar mit der Unterdrückung durch Könige assoziiert. Für die Poleis wiederum waren die Agone eine Möglichkeit, ihre Loyalität und Verbundenheit gegenüber Herrschern zum Ausdruck zu bringen und damit die Erwartung künftiger Wohltaten zu verbinden, ohne direkte Forderungen aufzustellen.

Ein weiteres Moment kommt hinzu. Ma betrachtet die imperialen Strategien hellenistischer Könige als Fortsetzung der Methoden des Achämenidenreiches, ${ }^{91}$ zu der auch die Förderung der dominanten ethnischen Gruppe gehörte. Die Förderung von Gymnasien durch die Könige bezeichnet er als „cultural Hellenism“,92 als Betonung der griechischen Dimension der Herrschaft. Dies gilt in noch stärkerem Maße für die großen Agone, die traditionell der Differenzmarkierung zwischen den Griechen und den anderen dienten. ${ }^{93}$ Durch die Neugründung von Agonen und durch die Verknüpfung von Agonen mit den Königen wurde in Asien und Ägypten betont, daß es sich um griechische Könige und demzufolge auch um griechisch geprägte Reiche handelte. Besonders deutlich wird dies in einer Siegerliste ptolemäischer Basileia von 267 v. Chr., die neben Disziplinen und Namen der Sieger auch deren Herkunft anführt. ${ }^{94}$ Hier finden sich sechs Thraker, vier

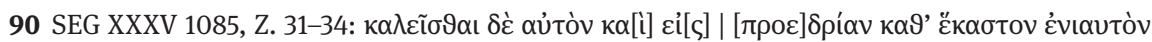

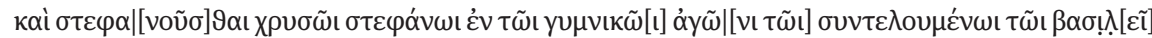
$\sum[\varepsilon \lambda \varepsilon \dot{\kappa} \omega \mathrm{t}$.

91 Ma 2003b, 179f. 191; Ma folgt hier den Arbeiten von Pierre Briant.

92 Ma 2003b, 188.

93 S. o. Anm. 39.

94 SEG XXVII 1114. Es handelt sich um eine schwarze Basaltstele unbekannter Herkunft, wohl aus dem Fayum. Editio princeps mit ausführlichem Kommentar bei Koenen 1977; s. auch Ebert 1979; Bingen 2007, 86-93; Pfeiffer 2015, 45-50 Nr. 8. 
Makedonen sowie jeweils ein Mann aus Thessalien, Boiotien, Samos, Halikarnassos, Tarent und Naukratis. Man darf aus dieser breiten Streuung nicht schließen, es habe sich um einen Agon mit panhellenischer Ausstrahlung gehandelt, der Teilnehmer aus der ganzen griechischen Welt anzog. Vielmehr können mehrere der in der Inschrift verzeichneten Sieger als Kleruchen identifiziert werden, die Land in der ägyptischen Chora besaßen. Sie hatten nicht das Bürgerrecht von Alexandria und griffen deshalb auf die alte Zugehörigkeit ihrer Familie zurück. ${ }^{95}$ Dadurch machten sie deutlich, daß ihre Wohnsitze in Ägypten keinesfalls ihre Zugehörigkeit zur griechischen Welt in Frage stellten, durch die Teilnahme an einem Agon, der auf den König bezogen war, drückten sie aber zugleich ihre Loyalität gegenüber dem Herrscher aus.

Der Inschrift kommt auch eine Schlüsselrolle in der Debatte zu, ob die ptolemäischen Basileia und die Ptolemaia auf die Hauptstadt Alexandria beschränkt waren oder ob sie auch in Form von lokalen Agonen in der ägyptischen Chora ausgetragen wurden. Letzteres wurde unter anderem von Koenen und PerpillouThomas vorgeschlagen; ${ }^{96}$ Thompson hat sogar die Hypothese in den Raum gestellt, es habe lokale Ausscheidungswettkämpfe gegeben, deren Sieger zu den großen Agonen nach Alexandria hätten fahren dürfen. ${ }^{97}$ Remijsen dagegen weist die Annahme von hellenistischen Agonen in der Chora zurück und verweist darauf, daß es sich beim Organisator der betreffenden Basileia von 267 v. Chr. um einen Bürger Alexandrias handelt; die Inschrift sei nur deshalb im Fayum und damit fern der Hauptstadt aufgestellt worden, weil der dortige Bewohner Amodokos drei Söhne unter den Siegern hatte. ${ }^{98}$ Allerdings müßte dann erklärt werden, warum die Liste keine Sieger aus Alexandria verzeichnet; bei einem Wettkampf in der Hauptstadt ist eine solche Dominanz von Athleten aus der Chora sehr unwahrscheinlich. Die Abhaltung von lokalen Ablegern der großen Agone Alexandrias bleibt die plausiblere, wenn auch bislang nicht beweisbare Annahme; träfe sie zu, hätten wir es hier gleichsam mit einer agonistischen Integration Ägyptens mit explizitem Bezug auf die Könige zu tun.

Bislang war in diesem Abschnitt von der Kommunikation zwischen Königen und den Poleis ihres Herrschaftsbereichs die Rede, doch sportliche Wettkämpfe spielten auch auf panhellenischer Ebene eine sehr wichtige Rolle. $\mathrm{Zu}$ nennen ist zunächst, daß Könige bisweilen die Agone der periodos besuchten oder sogar den

95 Koenen 1977, 23-28; s. auch Langenfeld 2009, 181; Remijsen 2014, 351.

96 Koenen 1977, 29-32; Ebert 1979, 4; Perpillou-Thomas 1993, 152-158.

97 Thompson 2000, 373. 386.

98 Remijsen 2009, 111 f.; ead. 2014, 354. 
Vorsitz führten; damit nutzten sie deren Rolle als panhellenische Plattform aus. ${ }^{99}$ Sie konnten aber auch neue Agone gründen und um deren Anerkennung in der griechischen Welt werben. Ein Schlaglicht darauf wirft das Nikuria-Dekret, in dem der Nesiotenbund die Ptolemaia in Alexandria, von Ptolemaios II. zu Ehren seines Vaters gegründet, als isolympischen Agon akzeptierte:

„[...] Da König Ptolemaios Soter vieler bedeutender Wohltaten Urheber war sowohl bei den Nesioten als auch bei den übrigen Griechen, indem er die Städte befreite, ihnen ihre Gesetze zurückgab, bei allen die traditionelle Verfassung einrichtete und Erleichterungen von Kontributionen gewährte, und da nun König Ptolemaios, nach der Übernahme der Königsherrschaft von seinem Vater, dasselbe Wohlwollen und dieselbe Fürsorge unentwegt erweist sowohl den Nesioten als auch den anderen Griechen, zu Ehren seines Vaters ein Opfer darbringt und einen isolympischen gymnischen, musischen und hippischen Agon ausrichtet und so den Göttern gegenüber seine Frömmigkeit beibehält und gegenüber seinen Vorfahren sein Wohlwollen, und da er appelliert an die Nesioten und die übrigen Griechen, zu beschließen, daß der Agon isolympisch sei, so gebührt es sich für alle Nesioten, die als erste Ptolemaios Soter mit gottgleichen Ehrungen geehrt haben sowohl aufgrund seiner allgemein allen erwiesenen Wohltaten als auch aufgrund seiner Einzelnen gewährten Hilfe, König Ptolemaios anläßlich seines Appells in sonstigen Belangen zu unterstützen und nun entsprechend seinem Vorhaben mit aller Bereitwilligkeit zu beschließen, [...] daß der Agon isolympisch sei, daß es für die Sieger, soweit sie Nesioten sind, dieselben Ehrungen geben soll, welche für jeden Nesioten in den Gesetzen für die Olympiasieger verzeichnet sind [...].“100

99 Die Nemeen beispielsweise wurden 315 v. Chr. von Kassander (Diod. 19,64,1), 223 v. Chr. von Antigonos Doson (Pol. 2,70,4f.) und 209 v. Chr. von Philipp V. geleitet (Liv. 27,30,17; vgl. Pol. 10,26,1); Philipp besuchte die Nemeen bereits 217 v. Chr. (Pol. 5,101,5f.); s. dazu Buraselis 2013, 171f. 183, und Freitag 2013, 142.

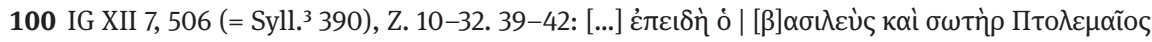

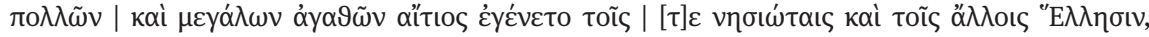

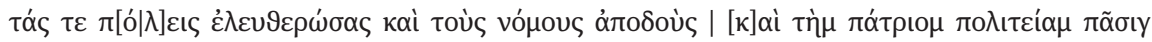

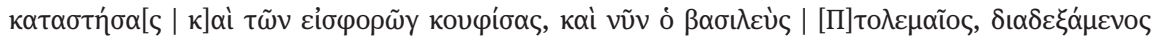

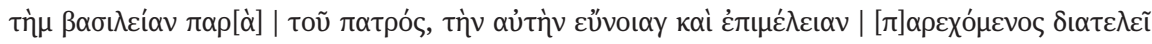

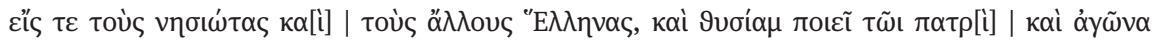

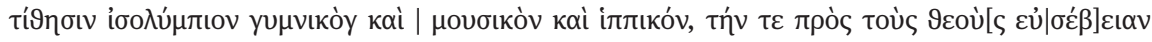

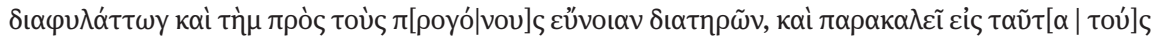

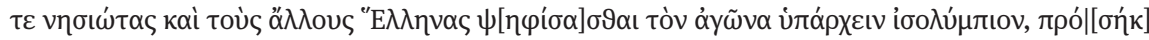

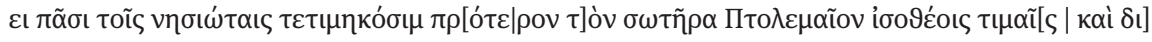

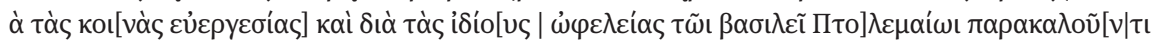

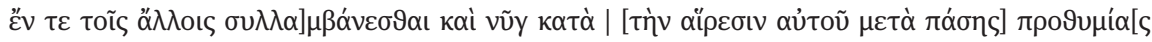

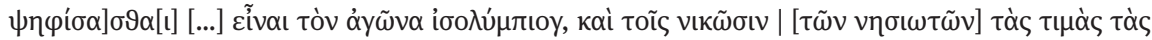

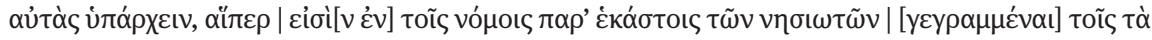

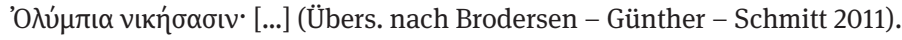


Seit dem Fund einer delphischen Inschrift ${ }^{101}$ ist die traditionelle Datierung, welche die ersten Ptolemaia im Winter 279/8 v. Chr. und das Nikuria-Dekret etwas früher, also 280/79 v. Chr., ansetzt, nicht mehr unumstritten, denn das Dekret der delphischen Amphiktyonie stimmt fast im Wortlaut überein, ist aber erst in den späten 260er Jahren entstanden. Hazzard postulierte eine zeitgleiche Entstehung beider Inschriften und datierte deshalb das Nikuria-Dekret, auch unter Verweis auf ägyptische Quellen, auf 263 v. Chr. herab. ${ }^{102}$ Doch damit konnte er sich nicht durchsetzen, die Mehrheit der Forschung hält mit guten Argumenten an der Datierung 280/79 v. Chr. fest. ${ }^{103}$ Schon Habicht hatte bald nach dem delphischen Neufund erwogen, daß Ptolemaios die Amphiktyonie aus politischen Gründen erst viel später als die Nesioten in seinen Aufruf einbezog. ${ }^{104}$

In hellenistischer Zeit wurden isolympische, aber auch isopythische und isonemeische Wettkämpfe populär, das Nikuria-Dekret enthält den frühesten Beleg für diese neue agonistische Kategorie. Worin die durch iso- ausgedrückte Gleichheit bestand, wird aus diesem und anderen Texten hinreichend deutlich: Es ging nicht um eine Übernahme der olympischen Disziplinen und Altersklassen - eine Auffassung, die in der älteren Forschung regelmäßig und auch heute noch vereinzelt $\mathrm{zu}$ finden ist ${ }^{105}$-, sondern um die Belohnungen für die Sieger. In griechischen Poleis gab es gesetzliche Regelungen zu den Prämien und Ehrungen für Athleten, die bei den Olympischen Spielen oder anderen großen Wettkämpfen gesiegt hatten, ${ }^{106}$ und isolympischer Status bedeutet, daß Siegern dieses Agons von ihrer Polis dasselbe zuteil werden solle wie Olympiasiegern. ${ }^{107}$ Daraus folgt aber, daß isolympischer Status nicht einfach durch die Ausrichter festgelegt werden kann, sondern von der griechischen Welt anerkannt werden muß, diplomatische Initiativen gehen der Einrichtung eines solchen Agons zwangsläufig voraus.

Dies ist nun allerdings ein revolutionärer Vorgang, der in der Forschung nicht in seiner ganzen Bedeutung erkannt wurde. Ma selbst räumt zwar mit Verweis

101 FD III 4:357.

102 Hazzard 2000, 47-58.

103 Hauben 2004, 40-44; s. auch Meadows 2013, 28. Zum Verhältnis der Ptolemaia zur berühmten Prozession (Athen. 5,297c-203b) s. Dunand 1981; Thompson 2000.

104 Habicht 1970, 258f. Sánchez (2001, 338-341) hat auf eine Besonderheit des delphischen Dekrets hingewiesen: Die Amphiktyonie erkennt die Ptolemaia nicht nur als isolympisch an, sondern will die Autorität Delphis auch einsetzen, um andere Griechen zum selben Schritt zu veranlassen.

105 Crowther 1989; Garcia Romero 2012, 67-71. Lediglich ein Text (FD III 3.215, Z. 10. 16. 25) enthält einen Verweis auf Altersklassen, aber auch dort werden Ehren genannt.

106 Z. B. IG I 131 (Athen, 5. Jh. v. Chr.); IEphesos 1415 (frühes 3. Jh. v. Chr.).

107 Slater - Summa 2006, 280 f.; Langenfeld 2009, 181 f.; Remijsen 2011, 104; Pleket 2014, 367. 
auf das Nikuria-Dekret ein, daß das Modell der „peer polity interaction“ dem Einfluß der Könige nicht ausreichend Rechnung trage, doch da er als Neuerung der Ptolemaia nicht den isolympischen Status erkennt, sondern von der viel weicheren Kategorie des panhellenischen Agons spricht, ${ }^{108}$ fehlt hier das wesentliche Element des Vorgangs: Ein neuer, noch nicht einmal begründeter Agon wird mit den altehrwürdigen Olympischen Spielen, die in der Vorstellung der Griechen bereits seit 776 v. Chr. ausgetragen wurden, ${ }^{109}$ auf einer zentralen Ebene gleichgesetzt. Ohne die Macht eines Monarchen hätte die neue Kategorie auch nicht entstehen können; die Nesioten begründen ihre Anerkennung des Status ja explizit mit den Leistungen der beiden Ptolemäer für ihren Bund, Leistungen, die in dieser Dimension überhaupt nur Monarchen erbringen konnten. Ptolemaios II. betrieb erheblichen diplomatischen Aufwand, um dem von ihm ins Leben gerufenen Agon isolympischen Status zu verleihen. Dies tat er wohl weniger aus Sorge um das materielle Wohlergehen der Athleten, sondern weil die Anerkennung des isolympischen Status zugleich eine Anerkennung seiner Leistungen und seiner hervorgehobenen Position in der griechischen Welt war. Er schrieb hier den Namen seiner Dynastie in das panhellenisch höchst bedeutsame Feld der Agonistik ein.

War die neue Kategorie aber einmal in der Welt, wurde sie von der griechischen Poliswelt übernommen. Zwar sind auch für die Folgezeit isolympische Initiativen von Königen überliefert, ${ }^{110}$ doch nun wird die Bitte um Anerkennung eines isolympischen, isopythischen oder isonemeischen Status zu einem regelmäßig anzutreffenden Element in der Diplomatie zwischen griechischen Poleis und Ethne. Das gleiche gilt für die Kategorie „stephanitisch“; eine Inschrift aus Magnesia am Mäander liefert ein beeindruckendes Zeugnis für den Aufwand, mit dem diese Polis im zweiten Anlauf eine Anerkennung ihres Artemis Leukophryene geweihten stephanitischen Agons erreichen wollte. Gesandte wurden bis nach Syrakus und Mesopotamien geschickt, die positiven Bescheide der einzelnen Zielstädte sorgfältig dokumentiert. ${ }^{111}$ Man kann an dieser Thematik, den Verhandlungen über den Status von Agonen, sowohl die Mechanismen der „peer

108 Ma 2003a, $25 \mathrm{f}$.

109 Daß dieses Gründungsdatum nicht historisch ist, sondern ein späteres Konstrukt, und regelmäßige bedeutende Agone in Olympia erst seit ca. 700 v. Chr. stattfanden (Mallwitz 1988), spielt in diesem Zusammenhang keine Rolle.

110 Der Attalide Eumenes II. forderte 183/82 v. Chr. die Anerkennung der Nikephoria von Pergamon als isolympischer Agon; die (selbstverständlich positiven) Antworten der delphischen Amphiktyonie und des Aitolischen Bundes sind epigraphisch erhalten: FD III 3:240; FD III 3:261. 111 IMagnesia 16; s. Ebert 1982; Rigsby 1996, 179-279; Thonemann 2007; van Nijf - Williamson 2016, 46-48 mit Appendix I. 
polity interaction“ als auch die Macht der Könige, neue Strukturen zu schaffen, exemplarisch ablesen.

\section{Fazit}

Die Könige übten, dies zeigt der zuletzt angesprochene Aspekt deutlich auf, erheblichen Einfluß auf die griechische Agonistik aus. Die neue Kategorisierung der Agone ging auf ihre Initiative zurück; nur ein Monarch konnte zum ersten Mal einen Agon ins Leben rufen, dessen Siegern die Heimatstadt dieselben Ehrungen und Privilegien verlieh wie Olympiasiegern. Wenn sie wollten, konnten die Könige neue Agone gründen, konnten durch Einsatz ihrer finanziellen Ressourcen Dominanz in den hippischen Disziplinen gewinnen und athletische Talente fördern, um von deren agonistischem Ruhm zu profitieren. Auch deswegen ist der Hellenismus innerhalb der antiken Sportgeschichte eine Epoche mit eigenem Charakter, nicht lediglich eine aus politischen Entwicklungen abgeleitete Kategorie. ${ }^{112}$ Allerdings hatte der Einfluß der Könige Grenzen: Aufgrund ihrer Einbindung in Götterfeste waren die Strukturen der griechischen Agonistik gegenüber fundamentalen Umwälzungen resistent, und weder die Ptolemaia noch andere von Königen gegründete Agone erreichten jemals auch nur annähernd das Renommee der Olympischen Spiele. ${ }^{113}$

Im Zentrum des Aufsatzes stand jedoch nicht der Einfluß der Könige auf die Agonistik, sondern die Bedeutung der Agonistik für die Könige. Auffallend sind hier insbesondere die Unterschiede zwischen den Dynastien: Die Ptolemäer legten, vor allem unter Ptolemaios II., eine enorme Aktivität in den Pferde- und Wagenrennen an den Tag und dominierten in diesen Disziplinen über Jahrzehnte; auch die Förderung von Talenten in den gymnischen Disziplinen scheint ihnen wichtig gewesen zu sein. Während auch die Attaliden einige bedeutende hippische Siege errangen - wie bei den Ptolemäern lassen sich bei der Repräsentation der Erfolge direkte Bezüge auf die Spezifika ihrer Herrschaft herstellen -, waren andere Dynastien auf diesem Gebiet passiv. Man kann diesen Verzicht verstehen, wenn man sportliche Erfolge nicht eindimensional als rundum positiv wahrgenommene Nachweise von Sieghaftigkeit betrachtet, sondern die Ambivalenz agonistischer Erfolge berücksichtigt.

112 Langenfeld 2009; Mann 2016.

113 Aus diesem Grund fehlt der Annahme, die isolympischen Spiele in Alexandria hätten das Heiligtum von Olympia geschwächt (Orth 2014, 571), die Basis. 
Die Förderung von Talenten in den gymnischen Disziplinen ist schwer zu messen, auch hier scheinen sich die Ptolemäer am meisten engagiert zu haben. Für den Seleukiden Antiochos III. dagegen ist in der Inschrift aus Teos ein kreativer und bislang singulärer Weg überliefert, den König am Ruhm agonistischer Erfolge teilhaben zu lassen. Man fand hier einen Weg, die Macht des Königs im Kommunikationsraum der Agonistik sichtbar $\mathrm{zu}$ machen, ohne direkte Herrschaft auszudrücken. Ähnliches gilt für die vielen Agone, die den Namen von Königen trugen, an deren Geburtstag ausgetragen wurden oder in anderer Form auf sie bezogen waren: Hier wurde die Sprache der Agonistik benutzt, um die Könige in die traditionellen Rituale der Polis $\mathrm{zu}$ integrieren und gleichermaßen dem königlichen Anspruch wie dem Selbstbewußtsein der Bürgerschaft gerecht zu werden.

Danksagung: Die hier vorgestellten Überlegungen entstanden im Kontext des Mannheimer DFG-Projekts „Die Selbstdarstellung hellenistischer Athleten: soziale Identitäten, politische Identitäten, ethnische Identitäten“ (2013-2016). Alexander Meeus und Sebastian Scharff danke ich für ihre kritische Lektüre einer früheren Version dieses Aufsatzes, Jennifer Goetz für Quellen- und Literaturrecherchen. Im Colloquium Classicum der Universität Frankfurt am Main durfte ich meine Gedanken im Juni 2017 vortragen und diskutieren, wofür ich zu Dank verpflichtet bin.

\section{Bibliographie}

Albanidis 2015: Ev. Albanidis, The Gymnasiarch Menas of Sestos and His Contribution to Training Youth During the 2nd cent. B.C., in: Ev. Albanidis - Chr. Faniopoulos (Hgg.), Sports in Education from Antiquity to Modern Times, Edessa 2015, 39-43.

Aneziri 2003: S. Aneziri, Die Vereine der Dionysischen Techniten im Kontext der hellenistischen Gesellschaft, Stuttgart 2003.

Austin - Bastianini: C. Austin - G. Bastianini, Posidippi Pellaei quae supersunt omnia, Mailand 2002.

Barbantani 2012: S. Barbantani, Hellenistic Epinician, in: P. Agócs - Chr. Carey - R. Rawles (Hgg.), Receiving the komos. Ancient \& Modern Receptions of the Victory Ode, London 2012, 37-55.

Barbantani 2014: S. Barbantani, „Attica in Syria“. Persian War Reenactments and Reassessments of the Greek-Asian Relationships: a Literary Point of View, Erga-Logoi 2, 2014, 21-91.

Bastianini - Gallazzi 2001: G. Bastianini - Cl. Gallazzi, Papiri dell'Università degli Studi di Milano VIII: Posidippo di Pella, Epigrammi (P. Mil. Vogl. VIII 309), in Zusammenarbeit mit C. Austin, Mailand 2001. 


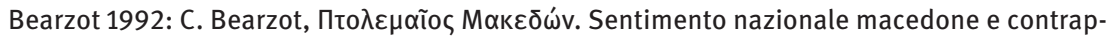
posizioni etniche all'inizio del regno tolemaico, in: M. Sordi (Hg.), Autoscienza e rappresentazione dei popoli nell'antichità, Mailand 1992, 39-53.

Bennett 2005: C. Bennett, Arsinoe and Berenice at the Olympics, ZPE 154, 2005, 91-96.

Bingen 2007: J. Bingen, Hellenistic Egypt. Monarchy, Society, Economy, Culture, hg. von R. S. Bagnall, Edinburgh 2007.

Blech 1982: M. Blech, Studien zum Kranz bei den Griechen, Berlin - New York 1982.

Brodersen - Günther - Schmitt 2011: K. Brodersen - W. Günther - H. H. Schmitt (Hgg.), Historische griechische Inschriften in Übersetzung, Darmstadt 2011.

Brunet 2003: St. Brunet, Olympic Hopefuls from Ephesos, Journal of Sport History 30, 2003, 219-235.

Bugh 2006: Gl. R. Bugh (Hg.), The Cambridge Companion to the Hellenistic World, Cambridge (UK) 2006.

Buraselis 1993: K. Buraselis, Ambivalent Roles of Centre and Periphery. Remarks on the Relation of the Cities of Greece with the Ptolemies until the End of Philometor's Age, in: P. Bilde u. a. (Hgg.), Centre and Periphery in the Hellenistic World, Aarhus 1993, 251-270.

Buraselis 2013: K. Buraselis, On the Nemean Games in the Hellenistic Period. The Vicissitudes of a Panhellenic Festival through War and Peace, in: N. Birgalias - K. Buraselis - P.

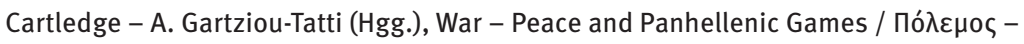

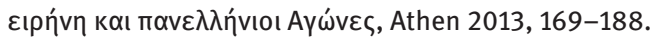

Carney 2013: E. Carney, Arsinoë of Egypt and Macedon. A Royal Life, Oxford 2013.

Chaniotis 2005: A. Chaniotis, Akzeptanz von Herrschaft durch ritualisierte Dankbarkeit und Erinnerung, in: C. Ambos - S. Hotz - G. Schwedler - St. Weinfurter (Hgg.), Die Welt der Rituale. Von der Antike bis heute, Darmstadt 2005, 188-204.

Chaniotis 2007: A. Chaniotis, La divinité mortelle d’Antiochos III à Téos, Kernos 20, 2007, 69-95.

Chankowski 2004: A. S. Chankowski, L'entraînement militaire des éphèbes dans les cités grecques d'Asie mineure à l'époque hellénistique. Nécessité pratique ou tradition atrophée, in: J.-Chr. Couvenhes - H. L. Fernoux (Hgg.), Les cités grecques et la guerre en Asie Mineure à l'époque hellénistique, Tours 2004, 55-76.

Chrubasik 2016: B. Chrubasik, Kings and Usurpers in the Seleukid Empire, Oxford 2016.

Clayman 2012: D. L. Clayman, Did Any Berenike Attend the Isthmian Games? A Literary Perspective on Posidippus 82 AB, ZPE 182, 2012, 121-130.

Crowther 1989: N. B. Crowther, The Sebastan Games in Naples (IvOl. 56), ZPE 79, 1989, 100-102.

Decker 1987: W. Decker, Sport und Spiel im Alten Ägypten, München 1987.

Debord 2003: P. Debord, Le culte royal chez les Séleucides, in: Fr. Prost (Hg.), L’Orient méditerranéen de la mort d’Alexandre aux campagnes de Pompée, Rennes 2003 , 281-309.

Di Nanni Durante 2015: D. Di Nanni Durante, Concorsi sportivi e propaganda politica in età ellenistica, Ariccia 2015.

Dunand 1981: Fr. Dunand, Fête et propagande à Alexandrie sous les Lagides, in: ders. (Hg.), La fête, pratique et discours: d’Alexandrie hellénistique à la mission de Besançon, Paris 1981, 13-40.

Ebert 1972: J. Ebert, Griechische Epigramme auf Sieger an gymnischen und hippischen Agonen, Berlin 1972.

Ebert 1979: J. Ebert, Zu Fackelläufen und anderen Problemen in einer griechischen agonistischen Inschrift aus Ägypten, Stadion 5, 1979, 1-19. 
Ebert 1982: J. Ebert, Zur Stiftungsurkunde der Leukophryena in Magnesia am Mäander (I.Magn. 16), Philologus 126, 1982, 198-216.

Erskine 2003: A. Erskine (Hg.), A Companion to the Hellenistic World, Oxford 2003.

Erskine 2013: A. Erskine, The View from the Old World. Contemporary Perspectives on Hellenistic Culture, in: E. Stavrianopoulou (Hg.), Shifting Social Imaginaries in the Hellenistic Period. Narrations, Practices, and Images, Leiden - Boston 2013, 339-363.

Freitag 2013: Kl. Freitag, Die panhellenischen Heiligtümer und die griechischen Bundesstaaten in hellenistischer Zeit, in: N. Birgalias - K. Buraselis - P. Cartledge - A. Gartziou-Tatti

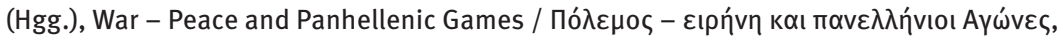
Athen 2013, 131-148.

Garcia Romero 2012: F. Garcia Romero, Sports Festivals Like the Olympic Games: Iso-Olympics, in: W. Petermandl - Chr. Ulf (Hgg.), Youth - Sport - Olympic Games, Hildesheim 2012, 59-72.

Gardiner 1930: N. E. Gardiner, Athletics of the Ancient World, Oxford 1930.

Gauthier 1993: Ph. Gauthier, Les cités héllenistiques, in: M. H. Hansen (Hg.), The Ancient Greek City-state, Kopenhagen 1993, 211-231.

Gehrke 2013: H.-J. Gehrke, The Victorious King. Reflections on the Hellenistic Monarchy, in: N. Luraghi (Hg.), The Splendors and Miseries of Ruling Alone. Encounters with Monarchy from Archaic Greece to the Hellenistic Mediterranean, Stuttgart 2013, 73-98 (= Der siegreiche König. Überlegungen zur hellenistischen Monarchie, AKG 64, 1982, 247-277).

Gotter 2013: U. Gotter, The Castrated King. or: The Everyday Monstrosity of Late Hellenistic Kingship, in: N. Luraghi (Hg.), The Splendors and Miseries of Ruling Alone. Encounters with Monarchy from Archaic Greece to the Hellenistic Mediterranean, Stuttgart 2013, 207-230.

Gruen 1993: E. S. Gruen, The Polis in the Hellenistic World, in: R. M. Rosen - J. Farrell (Hgg.), Nomodeiktes (FS Ostwald), Ann Arbor 1993, 339-354.

Harris 1972: H. A. Harris, Sport in Greece and Rome, Ithaca/NY 1972.

Habicht 1970: Chr. Habicht, Gottmenschentum und griechische Städte, München 1970.

Hauben 2004: H. Hauben, A Phoenician King in the Service of the Ptolemies. Philocles of Sidon Revisited, AncSoc 34, 2004, 27-44.

Hazzard 2000: R. A. Hazzard, Imagination of a Monarchy. Studies in Ptolemaic Propaganda, Toronto 2000.

Herrmann 1965: P. Herrmann, Antiochos der Grosse und Teos, Anadolu 9, 1965, 29-159.

Hose 2015: M. Hose, Hippika (71-88), in: B. Seidensticker - A. Stähli - A. Wessels (Hgg.), Der Neue Poseidipp. Text - Übersetzung - Kommentar, Darmstadt 2015, 283-317.

Huß 2008: W. Huß, Die Tochter Berenike oder die Schwiegertochter Berenike? Bemerkungen zu einigen Epigrammen des Poseidippos von Pella, ZPE 165, 2008, 55-57.

Kah 2004: D. Kah, Militärische Ausbildung im hellenistischen Gymnasion, in: ders. - Scholz 2004, 47-90.

Kah - Scholz 2004: D. Kah - P. Scholz (Hgg.), Das hellenistische Gymnasion, Berlin 2004.

Kainz 2016: L. Kainz, „We are the Best, We are One, and We are Greeks!“ Reflections on the Ptolemies' Participation in the agones, in: Mann - Remijsen - Scharff 2016, 331-353.

Kennell 2013: N. M. Kennell, Who were the Neoi?, in: P. Martzavou - N. Papazarkadas (Hgg.), Epigraphical Approaches to the Postclassical Polis. Fourth Century BC to Second Century AD, Oxford 2013, 217-232.

Kertész 2013: I. Kertész, The Role of Hellenistic Pergamon in the Ancient and Modern Olympic Movement, in: P. Mauritsch - Chr. Ulf (Hgg.), Kultur(en). Formen des Alltäglichen in der Antike (FS I. Weiler), Graz 2013, 817-827. 
Koenen 1977: L. Koenen, Eine agonistische Inschrift aus Ägypten und frühptolemäische Königsfeste, Meisenheim 1977.

Kotsidu 2000: H. Kotsidu, TIMH KAI $\triangle \mathrm{O} \equiv$ A. Ehrungen für hellenistische Herrscher im griechischen Mutterland und in Kleinasien unter besonderer Berücksichtigung der archäologischen Denkmäler, Berlin 2000.

Kralli 2013: I. Kralli, The Panhellenic Games in the Political Agenda of Hellenistic Leaders, in: N. Birgalias - K. Buraselis - P. Cartledge - A. Gartziou-Tatti (Hgg.), War - Peace and

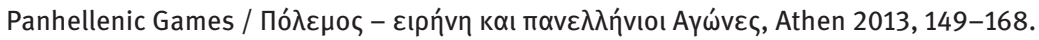

Kunze 2002: Chr. Kunze, Zum Greifen nah. Stilphänomene in der hellenistischen Skulptur und ihre inhaltliche Interpretation, München 2002.

Kurke 1991: L. Kurke, The Traffic in Praise. Pindar and the Poetics of Social Economy, Ithaca/NY 1991.

Kurke 1993: L. Kurke, The Economy of kudos, in: C. Dougherty - L. Kurke (Hrsg.), Cultural Poetics in Archaic Greece. Cult, Performance, Politics, Cambridge (UK) 1993, 131-163.

Kyle 2007: D. G. Kyle, Sport and Spectacle in the Ancient World, Malden 2007.

Langenfeld 2009: H. Langenfeld, Der Hellenismus als Epoche der Sportgeschichte, in: J.-F. Eckholdt - M. Sigismund - S. Sigismund (Hgg.), Geschehen und Gedächtnis. Die hellenistische Welt und ihre Wirkung (FS W. Orth), Berlin 2009, 177-199.

Lattmann 2012: Cl. Lattmann, Ritualisierter Siegpreis. Anmerkungen zur pragmatischen Dimension von Epinikien, Nikephoros 25, 2012, 19-78.

Ma 1999: J. Ma, Antiochos III and the Cities of Western Asia Minor, Oxford 1999.

Ma 2003a: J. Ma, Peer Polity Interaction in the Hellenistic Age, P \& P 180, 2003, 9-39.

Ma 2003b: J. Ma, Kings, in: Erskine 2003, 177-195.

Malay - Ricl 2009: H. Malay - M. Ricl, Two New Hellenistic Decrees from Aigai in Aiolis, EA 42, 2009, 39-60.

Mallwitz 1988: A. Mallwitz, Cult and Competition Locations at Olympia, in: W. J. Raschke (Hg.), The Archaeology of the Olympics, Madison 1988, 79-109.

Manieri 2009: A. Manieri, Agoni poetico-musicali nella Grecia antica I: Beozia (Testi e Commenti / Textes and Commentaries 25), Rom 2009.

Mann 2001: Chr. Mann, Athlet und Polis im archaischen und frühklassischen Griechenland, Göttingen 2001.

Mann 2016: Chr. Mann, Sport im Hellenismus: Forschungsstand und Forschungsperspektiven, in: Mann - Remijsen - Scharff 2016, 17-29.

Mann 2017: Chr. Mann, „He will win more competitions“. Talent promotion in ancient Greece, Journal of Olympic History 25, 2017, 48-53.

Mann - Remijsen - Scharff 2016: Chr. Mann - S. Remijsen - S. Scharff (Hgg.), Athletics in the Hellenistic World, Stuttgart 2016.

Matthaei - Zimmermann 2009: A. Matthaei - M. Zimmermann (Hgg.), Stadtbilder im Hellenismus, Berlin 2009.

Matthaei - Zimmermann 2014: A. Matthaei - M. Zimmermann (Hgg.), Stadtkultur im Hellenismus, Heidelberg 2014.

Matthaei - Zimmermann 2015: A. Matthaei - M. Zimmermann (Hgg.), Urbane Strukturen und bürgerliche Identität im Hellenismus, Heidelberg 2015.

Meadows 2013: A. Meadows, The Ptolemaic League of Islanders, in: K. Buraselis - M. Stefanou - D. J. Thompson (Hgg.), The Ptolemies, the Sea and the Nile, Cambridge (UK) 2013, 19-28.

Moretti 1957: L. Moretti, Olympionikai. I vincitori negli antichi agoni olimpici, Rom 1957. 
Moretti 1975: L. Moretti, Iscrizioni storiche ellenistiche. Volume II. Grecia centrale e settentrionale, Florenz 1975.

Müller 2009: S. Müller, Das hellenistische Königspaar in der medialen Repräsentation: Ptolemaios II. und Arsinoe II., Berlin u. a. 2009.

Nielsen 2007: Th. H. Nielsen, Olympia and the Classical Hellenic City-State Culture, Kopenhagen 2007.

Nielsen 2016: Th. H. Nielsen, Reflections on the Number of Athletic Festivals in Pre-Hellenistic Greece, in: Mann - Remijsen - Scharff 2016, 31-41.

Orth 2014: W. Orth, Der fromme Diadoche. Zur Situation der großen griechischen Heiligtümer um 300 v. Chr., in: H. Hauben - A. Meeus (Hgg.), The Age of the Successors and the Creation of the Hellenistic Kingdoms (323-276 B.C.), Leuven 2014, 559-575.

Perpillou-Thomas 1993: Fr. Perpillou-Thomas, Fêtes d’Égypte ptolémaïque et romaine d'après la documentation papyrologique grecque, Leuven 1993.

Pfeiffer 2015: St. Pfeiffer, Griechische und lateinische Inschriften zum Ptolemäerreich und zur römischen Provinz Aegyptus, Münster u. a. 2015.

Piejko 1991: F. Piejko, Antiochus III and Teos Reconsidered, Belleten. Türk Tarih Kurumu 55, 1991, 13-69.

Pleket 1975: H. W. Pleket, Games, Prizes, Athletes and Ideology. Some Aspects of the History of Sport in the Greco-Roman World, Stadion 1, 1975, 49-89.

Pleket 1978: H. W. Pleket, Sport und Leibesübungen in der griechischen Welt des hellenistischrömischen Zeitalters, in: H. Überhorst (Hg.), Geschichte der Leibesübungen. Bd. 2: Leibesübungen und Sport in der Antike: bei den frühen Reitervölkern, im klassischen Griechenland, bei den Etruskern, in der römischen Antike, in der hellenistischen Zeit, im Frühchristentum, Berlin 1978, 280-311.

Pleket 2001: H. W. Pleket, Zur Soziologie des antiken Sports, Nikephoros 14, 2001, 157-212.

Pleket 2005: H. W. Pleket, Athleten im Altertum. Soziale Herkunft und Ideologie, Nikephoros 18, 2005, 151-163.

Pleket 2014: H. W. Pleket, Sport in Hellenistic and Roman Asia Minor, in: P. Christesen - D. G. Kyle (Hgg.), A Companion to Sport and Spectacle in Greek and Roman Antiquity, Malden Oxford 2014, 364-375.

Remijsen 2009: S. Remijsen, Challenged by Egyptians. Greek Sports in the Third Century BC, in: Z. Papakonstantinou (Hg.), Sport in the Cultures of the Ancient World, London - New York 2009, 98-123.

Remijsen 2011: S. Remijsen, The So-Called „Crown-Games“. Terminology and Historical Context of the Ancient Categories for Agones, ZPE 177, 2011, 97-109.

Remijsen 2014: S. Remijsen, Greek Sport in Egypt: Status Symbol and Lifestyle, in: P. Christesen - D. G. Kyle (Hgg.), A Companion to Sport and Spectacle in Greek and Roman Antiquity, Malden - Oxford 2014, 349-363.

C. Renfrew - J. F. Cherry 1986: C. Renfrew - J. F. Cherry (Hgg.), Peer Polity Interaction and SocioPolitical Change, Cambridge (UK) 1986.

Rigsby 1996: K. J. Rigsby, Asylia: Territorial Inviolability in the Hellenistic World, Berkeley u.a. 1996.

Robert 1938: L. Robert, Études épigraphiques et philologiques, Paris 1938.

Robert 1969: L. Robert, Théophane de Mytilène a Constantinople, CRAI 1969, 42-64.

Robert 1984: L. Robert, Discours d'ouverture, in: Actes du VIIle Congrès international

d'épigraphie grecque et latine, 3-9 Octobre 1982, I, Athen 1984, 35-45.

Robert - Robert 1989: L. Robert - J. Robert, Claros I: Décrets hellénistiques, Paris 1989. 
Sánchez 2001: P. Sánchez, L’Amphictionie des Pyles et de Delphes: recherches sur son rôle historique, des origines au lle siècle de notre ère, Stuttgart 2001.

Scharff i. Dr.: S. Scharff, Victorious Kings. Hellenistic Rulers and Agonistic Success, in: M. Munn (Hg.), Hellenistic Monarchies in the Mediterranean World, Berlin - Boston i. Dr.

Schmitt 2005: H. H. Schmitt (Hg.), Lexikon des Hellenismus, Wiesbaden 2005.

Shear 2007: J. Shear, Royal Athenians. The Ptolemies and Attalids at the Panathenaia, in: 0. Palagia - A. Choremi-Spetsieri (Hgg.), The Panathenaic Games, Oxford 2007, 135-145.

Slater 2013: W. Slater, The Victor's Return, and the Categories of Games, in: P. Martzavou - N. Papazarkadas (Hgg.), Epigraphical Approaches to the Postclassical Polis. Fourth Century BC to Second Century AD, Oxford 2013, 139-163.

Slater - Summa 2006: W. J. Slater - D. Summa, Crowns at Magnesia, GRBS 46, 2006, 275-299. Thomas 1999: R. Thomas, Zu den Ringergruppen in der hellenistischen Kleinplastik, in: Hellenistische Gruppen (Gedenkschrift A. Linfert), Mainz 1999, 199-211.

Thompson 2000: D. J. Thompson, Philadelphus' Procession. Dynastic Power in a Mediterranean Context, in: L. Mooren (Hg.), Politics, Administration and Society in the Hellenistic and Roman World, Leuven 2000, 365-388.

Thompson 2005: D. J. Thompson, Posidippus, Poet of the Ptolemies, in: K. Gutzwiller (Hg.), The New Posidippus. A Hellenistic Poetry Book, New York 2005, 269-283.

Thonemann 2007: P. Thonemann, Magnesia and the Greeks of Asia (I.Magnesia 16.16), GRBS 47, 2007, 151-160.

Tracy - Habicht 1991: St. Tracy - Chr. Habicht, New and Old Panathenaic Victor Lists, Hesperia 60, 1991, 187-236.

Van Bremen 2007: R. Van Bremen, The Entire House is Full of Crowns. Hellenistic Agones and the Commemoration of Victory, in: S. Hornblower - C. Morgan (Hgg.), Pindar's Poetry, Patrons, and Festivals. From Archaic Greece to the Roman Empire, Oxford 2007, 345-375.

Van Nijf - Williamson 2016: O. Van Nijf - Chr. Williamson, Connecting the Greeks. Festival Networks in the Hellenistic World, in: Mann - Remijsen - Scharff (Hgg.), Athletics in the Hellenistic World, Stuttgart 2016, 43-71.

Wiemer 2017: H.-U. Wiemer, Siegen oder untergehen? Die hellenistische Monarchie in der neueren Forschung, in: St. Rebenich (Hg.), Monarchische Herrschaft im Altertum, Berlin Boston 2017, 305-339.

Wörrle 1988: M. Wörrle, Inschriften von Herakleia am Latmos I: Antiochos III., Zeuxis und Herakleia, Chiron 18, 1988, 421-476.

Wörrle - Zanker 1995: M. Wörrle - P. Zanker (Hgg.), Stadtbild und Bürgerbild im Hellenismus, München 1995. 